\title{
ASSOCIATIVITY OF THE REGULAR SEMIDIRECT PRODUCT OF EXISTENCE VARIETIES
}

\author{
BERND BILLHARDT and MÁRIA B. SZENDREI
}

(Received 6 March 1998; revised 17 February 2000)

Communicated by D. Easdown

\begin{abstract}
The associativity of the regular semidirect product of existence varieties introduced by Jones and Trotter was proved under certain conditions by Reilly and Zhang. Here we establish associativity in many new cases. Moreover, we prove that the regular semidirect product is right distributive with respect to the join operation. In particular, both associativity and right distributivity yield within the varieties of completely simple semigroups. Analogous results are obtained for e-pseudovarieties of finite regular semigroups.
\end{abstract}

2000 Mathematics subject classification: primary 20M17, $20 \mathrm{M} 07$.

\section{Introduction}

The wreath product and, more generally, the semidirect product constructions originate in group theory, but they have also been playing a central role in the theory of finite semigroups for a long time. Since a semidirect product or the wreath product of regular semigroups need not be regular, these constructions have been modified in the context of regular semigroups in several ways, see $[3,6,9]$. In the present paper, we deal with the product introduced in [9] by Jones and Trotter. They noticed that $\operatorname{Reg}(A * B)$, the set of all regular elements in a usual semidirect product $A * B$, is a (regular) subsemigroup in $A * B$ provided $A$ and $B$ are regular semigroups and one of them is completely simple. They introduced a partial operation-called the regular semidirect product - on the set of all e-varieties of regular semigroups as follows: if $\mathscr{U}$ and $\mathscr{V}$ are e-varieties such that $\mathscr{U}$ or $\mathscr{V} \subseteq \mathscr{C} \mathscr{S}$, the (e-)variety of all completely simple semigroups, then $\mathscr{U} * r \mathscr{V}$ is defined to be the e-variety generated by all semigroups

(c) 2000 Australian Mathematical Society $0263-6115 / 2000 \$ A 2.00+0.00$ 
$\operatorname{Reg}(A * B)$ with $A \in \mathscr{U}$ and $B \in \mathscr{V}$. Furthermore, they established a number of important properties of this product, and provided interesting decompositions of certain e-varieties, for example of $L \mathscr{I}$ and $\mathscr{E} \mathscr{S}$, the e-varieties of all locally inverse and of all $E$-solid semigroups.

The regular semidirect product of e-varieties is a generalization of the usual product of group varieties and is analogous to the semidirect product of pseudovarieties of finite semigroups. These classical products are well known to be associative (see $[4,13,14]$ ). The equality $\left(\mathscr{U} *_{r} \mathscr{V}\right) *_{r} \mathscr{W}=\mathscr{U} *_{r}\left(\mathscr{V} *_{r} \mathscr{W}\right)$ has been proved recently by Reilly and Zhang [15] in cases, where at least two of the e-varieties $\mathscr{U}, \mathscr{V}, \mathscr{W}$ are group varieties and some additional conditions are fulfilled.

The aim of this paper is to prove associativity under more general conditions. We establish the equality $\left(\mathscr{U} *_{r} \mathscr{V}\right) *_{r} \mathscr{W}=\mathscr{U} *_{r}\left(\mathscr{V} *_{r} \mathscr{W}\right)$ if either (i) $\mathscr{U} \subseteq \mathscr{C} \mathscr{S}, \mathscr{V}$ or $\mathscr{W} \subseteq \mathscr{C} \mathscr{S}$ and $\mathscr{V} *_{r} \mathscr{W} \subseteq L \mathscr{I}$ or $\mathscr{E} \mathscr{S}$, or (ii) $\mathscr{V}, \mathscr{W} \subseteq \mathscr{C} \mathscr{S}$.

Analogously, the semidirect product of e-pseudovarieties — the finitary analogues of e-varieties - can also be defined. Note that an interesting relationship is presented by Auinger and Trotter [1] between the usual semidirect product of certain pseudovarieties and the regular semidirect product of related e-pseudovarieties.

It turns out that our arguments carry over to e-pseudovarieties. What is more, in this context, 'overall' associativity is obtained in the sense that the equality $\left(\mathscr{U} *_{r} \mathscr{V}\right) *_{r} \mathscr{W}=\mathscr{U} *_{r}\left(\mathscr{V} *_{r} \mathscr{W}\right)$ is verified for any e-pseudovarieties $\mathscr{U}, \mathscr{V}, \mathscr{W}$, where both sides are defined, that is, for any e-pseudovarieties $\mathscr{U}, \mathscr{V}, \mathscr{W}$ such that at least two of them are e-pseudovarieties of completely simple semigroups.

In Section 1 we summarize the notions and results needed in the paper. The aim of Section 2 is to prove that if $\mathscr{U}, \mathscr{V}$ are e-varieties such that $\mathscr{U}$ or $\mathscr{V} \subseteq \mathscr{C} \mathscr{S}$ and $\mathscr{X}$ generates $\mathscr{U}$, then $\{X$ wrr $B: X \in \mathscr{X}, B \in \mathscr{V}\}$ generates $\mathscr{U} *_{r} \mathscr{V}$, where $X$ wrr $B$ stands for the subsemigroup of all regular elements of the usual wreath product of $X$ by $B$. As a corollary, we obtain that $\{(A$ wrr $B)$ wrr $C: A \in \mathscr{U}, B \in \mathscr{V}, C \in \mathscr{W}\}$ generates $\left(\mathscr{U} *_{r} \mathscr{V}\right) *_{r} \mathscr{W}$ provided at least two of the e-varieties $\mathscr{U}, \mathscr{V}, \mathscr{W}$ are contained in $\mathscr{C} \mathscr{S}$. Another corollary is the right distributivity of the regular semidirect product with respect to the join operation. Section 3 is devoted to proving that $\{A$ wrr $(B$ wrr $C): A \in \mathscr{U}, B \in \mathscr{V}, C \in \mathscr{W}\}$ generates $\mathscr{U} *_{r}\left(\mathscr{V} *_{r} \mathscr{W}\right)$ provided $\mathscr{U}, \mathscr{V}, \mathscr{W}$ are e-varieties such that at least two of them are contained in $\mathscr{C} \mathscr{S}$ and $\mathscr{V} *_{r} \mathscr{W} \subseteq L \mathscr{I}$ or $\mathscr{E} \mathscr{S}$. In Section 4 we establish the equality $\left(\mathscr{U} *_{r} \mathscr{V}\right) *_{r} \mathscr{W}=$ $\mathscr{U} *_{r}\left(\mathscr{V} *_{r} \mathscr{W}\right)$ in case (i) above and in cases, where either $\mathscr{V} \subseteq \mathscr{G} \cup \mathscr{L} \mathscr{Z}$ and $\mathscr{W} \subseteq \mathscr{C} \mathscr{S}$ or $\mathscr{V} \subseteq \mathscr{C} \mathscr{S}$ and $\mathscr{W} \subseteq \mathscr{G}$. (Here $\mathscr{G}$ and $\mathscr{L} \mathscr{Z}$ stand for the varieties of all groups and of all left zero semigrouns, respectively.) In Section 5 first we investigate the regular semidirect products, where one of the factors is a variety of rectangular bands. We show among others that, for every e-variety $\mathscr{V}$ with $\mathscr{V} \nsubseteq \mathscr{R} \mathscr{Z}$, we have $\mathscr{V} *_{r} \mathscr{R} \mathscr{B}=\mathscr{V} *_{r} \mathscr{R} \mathscr{Z}$, where $\mathscr{R} \mathscr{Z}$ and $\mathscr{R} \mathscr{B}$ denote the varieties of all right zero semigroups and of all rectangular bands, respectively. Moreover, we verify that the 
regular semidirect product of completely simple varieties is 'almost always' equal to a regular semidirect product, where one of the factors is a group variety. These two results allow us to obtain from the main result in Section 4 the general associativity result mentioned above. In the last section we summarize our results on e-pseudovarieties.

Associativity of another product of e-varieties based on a generalization of the $\lambda$-semidirect product to regular semigroups, where the second factor is locally $\mathscr{R}$ unipotent is investigated in [2].

\section{Preliminaries}

For the standard notions and notation in semigroup theory the reader is referred to [7]. A class of regular semigroups is termed an existence variety, or briefly an $e$-variety ([5], see also [11]) if it is closed under forming direct products, regular subsemigroups and homomorphic images. Note that a class of completely regular semigroups or that of inverse semigroups constitutes an e-variety if and only if it is a variety of unary semigroups in the usual sense when, for a completely regular semigroup, the unary operation maps each element to its inverse within the maximal subgroup containing it, and, for an inverse semigroup, the unary operation maps each element to its unique inverse. For example, the classes of all groups, all completely simple semigroups, all completely regular semigroups, all inverse semigroups, all orthodox semigroups, all locally inverse semigroups, all $E$-solid semigroups and all regular semigroups form e-varieties. We introduce notation for the following evarieties:

$\mathscr{T}$-trivial semigroups,

$\mathscr{L} \mathscr{Z}$-left zero semigroups,

$\mathscr{R} \mathscr{Z}$-right zero semigroups,

$\mathscr{R} \mathscr{B}$-rectangular bands,

$\mathscr{G}$-groups,

$\mathscr{L} \mathscr{G}$-left groups,

$\mathscr{R} \mathscr{G}$-right groups,

$\mathscr{C} \mathscr{S}$-completely simple semigroups,

$\mathscr{C} \mathscr{S}(\mathscr{V})$-completely simple semigroups whose subgroups belong to a group variety $\mathscr{V}$,

$\mathscr{C} \mathscr{R}$-completely regular semigroups,

$L \mathscr{I}$-locally inverse semigroups (that is, regular semigroups whose regular submonoids are inverse semigroups),

$L \mathscr{V}$-regular semigroups whose regular submonoids belong to an e-variety $\mathscr{V}$,

$\mathscr{E} \mathscr{S}-E$-solid semigroups (that is, regular semigroups whose subsemigroup generated by the idempotents is completely regular),

$\mathscr{R} \mathscr{S}$-regular semigroups. 
Let $\mathscr{C}$ be a class of regular semigroups. The e-variety generated by $\mathscr{C}$ is denoted by $\langle\mathscr{C}\rangle_{e}$. Denote by $\mathbf{P} \mathscr{C}, \mathbf{S}_{r} \mathscr{C}$ and $\mathbf{H} \mathscr{C}$ the classes consisting of all direct products of members of $\mathscr{C}$, of all regular subsemigroups of members of $\mathscr{C}$ and of all homomorphic images of members of $\mathscr{C}$, respectively. It is easy to see that $\mathbf{P S}, \mathscr{C} \subseteq \mathbf{S} r \mathbf{P} \mathscr{C}$ and

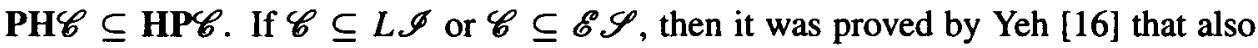
$\mathbf{S}_{r} \mathrm{H} \mathscr{C} \subseteq \mathrm{HS}_{r} \mathscr{C}$, and, as it is usual with varieties of algebras, we have $(\mathscr{C}\rangle_{e}=\mathrm{HS}_{r} \mathrm{P} \mathscr{C}$. However, the inclusion $\mathbf{S}_{r} \mathbf{H} \mathscr{C} \subseteq \mathbf{H S}_{r} \mathscr{C}$ does not hold for any class $\mathscr{C}$ of regular semigroups, and the class $\left(\mathbf{H S}_{r}\right)^{2} \mathscr{C}$ might be larger than $\mathrm{HS}_{r} \mathscr{C}$, see an example by Kadourek [10]. Using the terminology that a regular semigroup $T$ regularly divides a regular semigroup $S$ if $T$ is a homomorphic image of a regular subsemigroup of $S$, the latter fact can be expressed also in the way that regular divisibility is not a transitive relation. Put

$$
\begin{aligned}
\mathbf{D}^{\infty} \mathscr{C}= & \left\{S \in \mathscr{R} \mathscr{S}: \text { there exist } S_{0}, S_{1}, \ldots, S_{k} \in \mathscr{R} \mathscr{S}\right. \text { such that } \\
& \left.S_{0} \in \mathscr{C}, S_{k}=S \text { and } S_{i+1} \text { regularly divides } S_{i}(i=0,1, \ldots k-1)\right\} .
\end{aligned}
$$

Clearly, we have $\mathbf{D}^{\infty} \mathscr{C}=\bigcup_{k=0}^{\infty}\left(\mathbf{H S}_{r}\right)^{k} \mathscr{C}$ and $\mathbf{H D}^{\infty} \mathscr{C}=\mathbf{S}_{r} \mathbf{D}^{\infty} \mathscr{C}=\mathbf{D}^{\infty} \mathbf{H} \mathscr{C}=$ $\mathbf{D}^{\infty} \mathbf{S}_{r} \mathscr{C}=\mathbf{D}^{\infty} \mathscr{C}$.

The operator $\mathbf{P}$ allows us to form the direct product of any set of members in the given class. The above inclusions containing $\mathbf{P}$ do not imply the inclusion $\mathbf{P D}^{\infty} \mathscr{C} \subseteq$ $\mathbf{D}^{\infty} \mathbf{P} \mathscr{C}$. Hence we have to iterate the operators $\mathbf{D}^{\infty}$ and $\mathbf{P}$ transfinitely in order to get $\langle\mathscr{C}\rangle_{e}$ from $\mathscr{C}$. More precisely, we obtain $\langle\mathscr{C}\rangle_{e}$ as the union $\mathbf{E} \mathscr{C}$ of the following transfinite sequence $\mathbf{E}_{i} \mathscr{E}$ ( $i$ is an ordinal):

$$
\mathbf{E}_{0} \mathscr{C}=\mathscr{C}
$$

and

$$
\mathbf{E}_{i} \mathscr{C}= \begin{cases}\mathbf{D}^{\infty} \mathbf{P}\left(\mathbf{E}_{k} \mathscr{C}\right) & \text { if } i=k+1 \text { for some ordinal } k \\ \bigcup_{k<i} \mathbf{E}_{k} \mathscr{C} & \text { if } i \text { is a limit ordinal. }\end{cases}
$$

For, it is routine to verify that $\mathbf{E} \mathscr{C}$ is closed under the operations $\mathbf{P}, \mathbf{S}_{r}$ and $\mathbf{H}$, and so it is an e-variety. Furthermore, an easy transfinite induction shows that, for every ordinal $i, \mathbf{E}_{i} \mathscr{C}$ is contained in any e-variety containing $\mathscr{C}$, and so the same is true for $\mathbf{E} \mathscr{C}$.

For simplicity, we consider every class of regular semigroups as an abstract class, that is, we suppose that every class contains each isomorphic copy of its members.

If $S$ and $T$ are semigroups, then by writing $T \leq S$ we mean that $T$ is a subsemigroup in $S$. If $T$ is a regular subsemigroup in $S$, then we write that $T \leq r S$. The set of all regular elements of a semigroup $S$ is denoted by $\operatorname{Reg}(S)$.

As usual, we denote by $S^{1}$ the smallest monoid containing $S$. Then $S^{1}$ is equal to $S$ if $S$ has an identity element $1_{S}$, and $S=S \cup\left\{1_{S}\right\}$ with $1_{S} \notin S$ otherwise. If $T \leq S$ 
and $T$ has no identity, then we choose $1_{T}$ to be equal to $1_{S}$. So, for any semigroups $S, T$ with $T \leq S$, we have $T^{1} \leq S^{1}$.

If $S, T$ are semigroups and $\phi: S \rightarrow T$ is a surjective homomorphism, then $\phi$ can be uniquely extended to a surjective monoid homomorphism $S^{1} \rightarrow T^{1}$, which we denote by $\phi^{1}$. For, if $S$ has an identity, then surjectivity of $\phi$ ensures that $1_{S} \phi$ is an identity in $T$, that is, $1_{S} \phi=1_{T}$ and $\phi$ is a monoid homomorphism. So $\phi^{1}=\phi$. If $S$ has no identity, then define $1_{S} \phi^{1}=1_{T}$.

If $A, B$ are non-empty sets, then the set of all mappings of $A$ into $B$ is denoted by $B^{A}$. If $B$ is a semigroup, then $B^{A}$ stands for the direct power of $B$ to the exponent $A$, and the product of the elements $\alpha, \beta$ in the semigroup $B^{A}$ is denoted by $\alpha \beta$ or $\alpha \cdot \beta$. In contrast, if $\alpha \in B^{A}$ and $\beta \in C^{B}$ for some sets $A, B, C$, then their composition is denoted by $\alpha \circ \beta$.

For any $\alpha \in B^{A}$, the equivalence relation on $A$ induced by $\alpha$ is denoted by ker $\alpha$. Suppose now that $\alpha \in B^{A}$ is surjective. If $\beta \in C^{B}$ is any mapping, then $\alpha \circ \beta \in C^{A}$ and $\operatorname{ker}(\alpha \circ \beta) \supseteq \operatorname{ker} \alpha$. Conversely, for every mapping $\xi \in C^{A}$ with $\operatorname{ker} \xi \supseteq \operatorname{ker} \alpha$, there exists a unique mapping $\eta \in C^{B}$ such that $\xi=\alpha \circ \eta$. This $\eta$ is denoted by $\xi_{\alpha}$.

Let $A$ and $B$ be semigroups. Denote by End $A$ the endomorphism monoid of $A$. By a (left) action of $B$ on $A$ we mean an antihomomorphism $\varepsilon: B \rightarrow$ End $A, t \mapsto \varepsilon_{t}$. For brevity, we denote $a \varepsilon_{t}$ by ${ }^{t} a(a \in A, t \in B$ ). If $B$ is a monoid, then this action is said to be left unitary if $\varepsilon_{1_{B}}$ is the identity automorphism, or, equivalently, if ${ }^{1_{B}} a=a$ for every $a \in A$. The semidirect product $A * B$ of $A$ by $B$ with respect to this action is defined on the set $A \times B$ by the multiplication

$$
(a, t)(b, u)=(a \cdot b, t u) \quad(a, b \in A, t, u \in B)
$$

A straightforward calculation shows that $A * B$ is a semigroup.

The wreath product of a semigroup $A$ by a semigroup $B$ is the semidirect product of the direct power $A^{B^{1}}$ of $A$ by $B$ with respect to the following action: for every $\alpha \in A^{B^{\prime}}$ and $t \in B$, we define ${ }^{\prime} \alpha \in A^{B^{\prime}}$ by $x\left({ }^{\prime} \alpha\right)=(x t) \alpha\left(x \in B^{1}\right)$. Notice that this action is left unitary provided $B$ has an identity. The wreath product of $A$ by $B$ is denoted by $A$ wr $B$.

There is a natural embedding of any semidirect product $A * B$, where the action of $B$ on $A$ is left unitary provided $B$ has an identity, into $A$ wr $B$ :

$$
v: A * B \rightarrow A \text { wr } B,(a, b) v=(\alpha, b), \text { where } \alpha: B^{1} \rightarrow A, x \alpha={ }^{x} a\left(x \in B^{1}\right) \text {. }
$$

A semidirect or the wreath product of regular semigroups need not be regular. However, Jones and Trotter [9] introduced a regular version of the semidirect product, and initiated the study of the product of e-varieties induced by this product. They noticed that if $A$ and $B$ are regular semigroups, $B$ acts on $A$ and at least one of $A$ and $B$ is completely simple, then $\operatorname{Reg}(A * B)$ forms a (regular) subsemigroup 
in $A * B$, and they defined the regular semidirect product $A *_{r} B$ of $A$ by $B$ to be $\operatorname{Reg}(A * B)$. In particular, $\operatorname{Reg}(A \operatorname{wr} B)$ is a regular subsemigroup in $A$ wr $B$, which we denote by $A$ wrr $B$. Since a homomorphism maps regular elements to regular ones, we have $(\operatorname{Reg}(A * B)) \nu \subseteq \operatorname{Reg}(A$ wr $B)$ for the natural embedding $\nu$, and so $A *_{r} B$ is embeddable into $A$ wrr $B$ as long as the action of $B$ on $A$ is left unitary provided $B$ has an identity.

Given two e-varieties $\mathscr{U}, \mathscr{V}$ such that $\mathscr{U}$ or $\mathscr{V} \subseteq \mathscr{C} \mathscr{S}$, their regular semidirect product is defined in [9] as follows:

$$
\begin{aligned}
\mathscr{U} *_{r} \mathscr{V}= & \left\langle A *_{r} B: A \in \mathscr{U}, B \in \mathscr{V} \text { and } B \text { acts on } A\right. \text { such that } \\
& \text { the action is left unitary provided } B \text { has an identity }\rangle_{e} .
\end{aligned}
$$

Since each $A *_{r} B$ in the generating set is embeddable into $A$ wrr $B$, we obtain the following result.

RESULT 1.1. If $\mathscr{U}$ and $\mathscr{V}$ are e-varieties such that $\mathscr{U}$ or $\mathscr{V} \subseteq \mathscr{C} \mathscr{S}$, then we have $\mathscr{U} *_{r} \mathscr{V}=\langle A \text { wrr } B: A \in \mathscr{U}, B \in \mathscr{V}\rangle_{e}$.

We also need the following results from [9].

RESULT 1.2. Let $\mathscr{V}, \mathscr{W}$ be e-varieties such that $\mathscr{V}$ or $\mathscr{W} \subseteq \mathscr{C} \mathscr{S}$.

(i) We have $\mathscr{V} *_{r} \mathscr{W} \subseteq L \mathscr{I}$ if and only if either

$$
\mathscr{V} \subseteq L \mathscr{I} \text { and } \mathscr{W} \subseteq \mathscr{C} \mathscr{S}
$$

or

$$
\mathscr{V} \subseteq \mathscr{R} \mathscr{B} \text { and } \mathscr{W} \subseteq L \mathscr{I}
$$

(ii) We have $\mathscr{V} *_{r} \mathscr{W} \subseteq \mathscr{E} \mathscr{S}$ if and only if either

$$
\mathscr{V} \subseteq \mathscr{C} \mathscr{S} \text { and } \mathscr{W} \subseteq \mathscr{E} \mathscr{S}
$$

or

$$
\mathscr{V} \subseteq \mathscr{E} \mathscr{S} \text { and } \mathscr{W} \subseteq \mathscr{L} \mathscr{G}
$$

RESULT 1.3. Let $A, B$ be regular semigroups such that $A \in \mathscr{C} \mathscr{S}$ and $B$ acts on $A$. Then $(A * B) \cdot E(A * B) \subseteq \operatorname{Reg}(A * B)$.

RESULT 1.4. Let $\mathscr{V}$ be any e-variety. Then we have

(i) $\mathscr{V} *_{r} \mathscr{B} \mathscr{B} \subseteq L \mathscr{V}$,

(ii) $\mathscr{L} \mathscr{Z} \subseteq \mathscr{V} *_{r} \mathscr{R} \mathscr{Z}$ if $\mathscr{V} \nsubseteq \mathscr{R} \mathscr{Z}$, and

(iii) $\mathscr{C} \mathscr{S}(\mathscr{V})=\mathscr{V} *_{r} \mathscr{R} \mathscr{Z}$ if $\mathscr{T} \neq \mathscr{V} \subseteq \mathscr{G}$. 
RESULT 1.5. For any e-varieties $\mathscr{V}, \mathscr{W}$ with $\mathscr{V}, \mathscr{W} \subseteq \mathscr{C} \mathscr{S}$, we have $\mathscr{V} *_{r} \mathscr{W} \subseteq \mathscr{C} \mathscr{S}$.

Since every semidirect product of a left zero semigroup by another semigroup is isomorphic to their direct product, we get the following result, see [15].

RESULT 1.6. For any e-variety $\mathscr{V}$, we have $\mathscr{L} \mathscr{Z} *_{r} \mathscr{V}=\mathscr{L} \mathscr{Z} \vee \mathscr{V}$.

\section{Generating sets of $\mathscr{U} *_{r} \mathscr{V}$ via those of $\mathscr{U}$}

First we determine the regular elements of a semidirect product of regular semigroups. This lemma plays a crucial role throughout the paper. In the main result of the section, we prove that if $\mathscr{U}, \mathscr{V}$ are e-varieties such that $\mathscr{U}$ or $\mathscr{V} \subseteq \mathscr{C} \mathscr{S}$, and $\mathscr{X} \subseteq \mathscr{U}$ such that $\mathscr{U}=\langle\mathscr{X}\rangle_{e}$, then $\mathscr{U} *_{r} \mathscr{V}=\langle X \text { wrr } B: X \in \mathscr{X}, B \in \mathscr{V}\rangle_{e}$. As a consequence, we obtain that $\{(A$ wrr $B)$ wrr $C: A \in \mathscr{U}, B \in \mathscr{V}, C \in \mathscr{W}\}$ generates the e-variety $\left(\mathscr{U} *_{r} \mathscr{V}\right) *_{r} \mathscr{W}$ provided at least two of the e-varieties $\mathscr{U}, \mathscr{V}, \mathscr{W}$ are in $\mathscr{C} \mathscr{S}$. Moreover, we establish that $\left(\bigvee_{i \in I} \mathscr{U}_{i}\right) *_{r} \mathscr{V}=\bigvee_{i \in I}\left(\mathscr{U}_{i} *_{r} \mathscr{V}\right)$ for any e-varieties $\mathscr{U}_{i}(i \in I)$ and $\mathscr{V}$ if either $\mathscr{U}_{i} \subseteq \mathscr{C} \mathscr{S}$ for each $i \in I$ or $\mathscr{V} \subseteq \mathscr{C} \mathscr{S}$.

The regular elements in a semidirect product of regular semigroups can be described in the following way.

\section{LEMMA 2.1. Let $A$ and $B$ be regular semigroups and let $B$ act on $A$. Then}

$$
\operatorname{Reg}(A * B)=\left\{(a, b) \in A * B: \text { there exists } b^{\prime} \in V(b) \text { such that }{ }^{b b^{\prime}} a \geq \mathscr{L} \text { a in } A\right\} .
$$

Moreover, if $(a, b) \in A * B, x \in V(a)$ and $b^{\prime} \in V(b)$ such that ${ }^{b b^{\prime}} a \geq \mathscr{L} a$, then $\left({ }^{b} x, b^{\prime}\right) \in V((a, b))$.

PROOF. Suppose first that $(a, b) \in \operatorname{Reg}(A * B)$. Then there exists $\left(a^{\prime}, b^{\prime}\right) \in$ $V((a, b))$, that is, there exist $a^{\prime} \in A$ and $b^{\prime} \in B$ such that $(a, b)\left(a^{\prime}, b^{\prime}\right)(a, b)=(a, b)$ and $\left(a^{\prime}, b^{\prime}\right)(a, b)\left(a^{\prime}, b^{\prime}\right)=\left(a^{\prime}, b^{\prime}\right)$ hold. This implies that $b^{\prime} \in V(b)$ and $a \cdot{ }^{b} a^{\prime} \cdot b b^{\prime} a=a$. Hence it is immediate that $a \in A \cdot{ }^{b b^{\prime}} a$, and so $a \leq \mathscr{L}^{b b^{\prime}} a$.

The reverse inclusion follows if we prove the last statement. Suppose that $(a, b) \in$ $A * B, x \in V(a)$ and $b^{\prime} \in V(b)$ such that ${ }^{b b^{\prime}} a \geq \mathscr{L} a$. It is obvious that ${ }^{b b^{\prime}} x \in V\left({ }^{b b^{\prime}} a\right)$ also holds, and so ${ }^{b b^{\prime}} x \cdot{ }^{b b^{\prime}} a$ is an idempotent $\mathscr{L}$-related to ${ }^{b b^{\prime}} a$. Therefore, ${ }^{b b^{\prime}} a \geq \mathscr{L} a$ implies $a \cdot{ }^{b b^{\prime}} x \cdot{ }^{b b^{\prime}} a=a$, whence $(a, b)\left({ }^{b^{\prime}} x, b^{\prime}\right)(a, b)=(a, b)$. Since ${ }^{b^{\prime}} x \cdot b^{\prime} a \cdot b^{\prime} b b_{x}^{\prime}=$ $b^{\prime}\left(b^{b^{\prime}} x \cdot{ }^{b b^{\prime}} a \cdot{ }^{b b^{\prime}} x\right)={ }^{b^{\prime}}\left(b^{b} x\right)={ }^{b^{\prime}} x$, we also have $\left({ }^{b^{\prime}} x, b^{\prime}\right)(a, b)\left({ }^{b^{\prime}} x, b^{\prime}\right)=\left({ }^{b^{\prime}} x, b^{\prime}\right)$, proving that $\left(b^{\prime} x, b^{\prime}\right) \in V((a, b))$.

Now we intend to show that if a regular semigroup $Q$ is obtained from another one, say $A$ [from other ones, say $A_{i}(i \in I)$ ] by forming a regular subsemigroup or a homomorphic image [or the direct product], then $Q$ wrr $B$ is obtained from $A$ wrr $B$ [from $A_{i}$ wrr $B(i \in I)$ ] by making use of the same operator. 
LEMMA 2.2. Let $A, A^{\prime}, B$ be semigroups such that $A^{\prime} \leq A$. Then $A^{\prime}$ wr $B \leq$ $A$ wr $B$.

PROOF. This is obvious.

LEMMA 2.3. Let $A, A^{\prime}, B$ be regular semigroups such that $A^{\prime} \leq r A$ and $A$ or $B \in \mathscr{C} \mathscr{S}$. Then $A^{\prime}$ wrr $B \leq_{r} A$ wrr $B$.

ProOF. By Lemma 2.2, we have $A^{\prime}$ wrr $B=\operatorname{Reg}\left(A^{\prime}\right.$ wr $\left.B\right) \leq A^{\prime}$ wr $B \leq A$ wr $B$, and so $A^{\prime}$ wrr $B \leq_{r} \operatorname{Reg}(A$ wr $B)=A$ wrr $B$.

LEMMA 2.4. Let $A, \bar{A}, B$ be semigroups, and let $\phi: A \rightarrow \bar{A}$ be a surjective homomorphism. Then the mapping $\Phi: A \mathrm{wr} B \rightarrow \bar{A} \mathrm{wr} B$ defined by $(\alpha, b) \Phi=$ $(\alpha \circ \phi, b)$ is a surjective homomorphism.

PROOF. Straightforward.

LEMMA 2.5. Let $A, \bar{A}, B$ be regular semigroups, where $A$ or $B \in \mathscr{C} \mathscr{S}$. Let $\phi$ : $A \rightarrow \bar{A}$ be a surjective homomorphism. Then the mapping $\Phi_{r}: A$ wrr $B \rightarrow \bar{A}$ wrr $B$ defined by $(\alpha, b) \Phi_{r}=(\alpha \circ \phi, b)$ is a surjective homomorphism.

PROOF. The mapping $\Phi_{r}$ considered here is the restriction to regular elements of the homomorphism $\Phi$ defined in Lemma 2.4. Therefore, all we have to show is that if $(\bar{\alpha}, b) \in \operatorname{Reg}(\bar{A}$ wr $B)$, then there exists $\alpha \in A^{B^{1}}$ such that $\bar{\alpha}=\alpha \circ \phi$ and $(\alpha, b) \in \operatorname{Reg}(A$ wr $B)$.

Let $(\bar{\alpha}, b) \in \operatorname{Reg}(\bar{A}$ wr $B)$. Then Lemma 2.1 shows that there exists $b^{\prime} \in V(b)$ such that ${ }^{b b^{\prime}} \bar{\alpha} \geq \mathscr{L} \bar{\alpha}$ in $\bar{A}^{B^{\prime}}$, that is, $\left(x b b^{\prime}\right) \bar{\alpha} \geq \mathscr{L} x \bar{\alpha}$ in $\bar{A}$ for any $x \in B^{1}$. For every $\bar{a} \in \bar{A}$, let us choose and fix an inverse $\bar{a}^{\prime}$ of $\bar{a}$ in $\bar{A}$. Since $A$ is regular and $\phi$ is a surjective homomorphism, for each $\bar{a} \in \bar{A}$, there exist mutual inverse elements $r_{\bar{a}}, r_{\bar{a}}^{\prime}$ in $A$ such that $r_{\bar{a}} \phi=\bar{a}$ and $r_{\bar{a}}^{\prime} \phi=\bar{a}^{\prime}$. Let us fix such a pair for each $\bar{a} \in \bar{A}$. Define $\alpha \in A^{B^{\prime}}$ in such a manner that

$$
x \alpha=r_{x \bar{\alpha}} \cdot r_{\left(x b b^{\prime}\right) \bar{\alpha}}^{\prime} \cdot r_{\left(x b b^{\prime}\right) \bar{\alpha}}
$$

for every $x \in B^{1}$. Then $\left(x b b^{\prime}\right) \alpha=r_{\left(x b b^{\prime}\right) \bar{\alpha}}$ for each $x \in B^{1}$, and hence ${ }^{b b^{\prime}} \alpha \geq \mathscr{L}^{\alpha} \alpha$ easily follows. Thus $(\alpha, b) \in \operatorname{Reg}(A$ wr $B)$. Moreover, for every $x \in B^{1}$, we have

$$
x(\alpha \circ \phi)=(x \alpha) \phi=\left(r_{x \bar{\alpha}} \cdot r_{\left(x \dot{b} b^{\prime}\right) \bar{\alpha}}^{\prime} \cdot r_{\left(x b b^{\prime}\right) \bar{\alpha}}\right) \phi=x \bar{\alpha} \cdot\left(\left(x b b^{\prime}\right) \bar{\alpha}\right)^{\prime} \cdot\left(x b b^{\prime}\right) \bar{\alpha},
$$

where $\bar{e}=\left(\left(x b b^{\prime}\right) \bar{\alpha}\right)^{\prime} \cdot\left(x b b^{\prime}\right) \bar{\alpha}$ is an idempotent $\mathscr{L}$-related to $\left(x b b^{\prime}\right) \bar{\alpha}$. Since $\left(x b b^{\prime}\right) \bar{\alpha} \geq \mathscr{L} x \bar{\alpha}$, we infer that $x \bar{\alpha} \cdot \bar{e}=x \bar{\alpha}$, proving that $x(\alpha \circ \phi)=x \bar{\alpha}\left(x \in B^{1}\right)$, that is, $\alpha \circ \phi=\bar{\alpha}$. The proof is complete. 
LEMMA 2.6. Let $A_{i}(i \in I)$ and $B$ be semigroups. Define a mapping

$$
\Phi:\left(\prod_{i \in I} A_{i}\right) \text { wr } B \rightarrow \prod_{i \in I}\left(A_{i} \text { wr } B\right)
$$

as follows: for every $(\alpha, b) \in\left(\prod_{i \in I} A_{i}\right)$ wr $B$, let $(\alpha, b) \Phi$ be $F \in \prod_{i \in I}\left(A_{i}\right.$ wr $\left.B\right)$, where, for each $i \in I, i F=\left(i F_{1}, i F_{2}\right) \in A_{i}$ wr $B$ such that $i F_{2}=b$ and $x\left(i F_{1}\right)=$ $i(x \alpha)$ for any $x \in B^{1}$. Then $\Phi$ is an embedding.

Proof. Let $(\alpha, b),(\beta, c) \in\left(\prod_{i \in l} A_{i}\right)$ wr $B$, and let $(\alpha, b) \Phi=F,(\beta, c) \Phi=G$. In order to show that $\Phi$ is injective, suppose that $F=G$. Then $b=i F_{2}=i G_{2}=c$ and $i(x \alpha)=x\left(i F_{1}\right)=x\left(i G_{1}\right)=i(x \beta)$ are valid for every $i \in I$ and $x \in B^{1}$. Thus we see that $b=c$ and $\alpha=\beta$ which was to be shown.

In order to verify that $\Phi$ is a homomorphism, we have to prove that $((\alpha, b)(\beta, c)) \Phi=$ $F G$. By definition, we have

$$
(\alpha, b)(\beta, c)=\left(\alpha \cdot{ }^{b} \beta, b c\right)
$$

and, for every $i \in I$, we obtain that

$$
\begin{aligned}
\left(i(F G)_{1}, i(F G)_{2}\right) & =i(F G)=i F \cdot i G=\left(i F_{1}, i F_{2}\right)\left(i G_{1}, i G_{2}\right) \\
& =\left(i F_{1} \cdot{ }^{i F_{2}}\left(i G_{1}\right), i F_{2} \cdot i G_{2}\right)
\end{aligned}
$$

However, since $(\alpha, b) \Phi=F$ and $(\beta, c) \Phi=G$, we have

$$
i F_{2}=b, x\left(i F_{1}\right)=i(x \alpha) \text { and } i G_{2}=c, x\left(i G_{1}\right)=i(x \beta)
$$

for every $i \in I$ and $x \in B^{1}$. Thus $b c=i F_{2} \cdot i G_{2}=i(F G)_{2}$ easily follows. Moreover, for any $i \in I$ and $x \in B^{1}$, we infer that

$$
\begin{aligned}
i\left(x\left(\alpha \cdot{ }^{b} \beta\right)\right) & =i(x \alpha \cdot(x b) \beta)=i(x \alpha) \cdot i((x b) \beta)=x\left(i F_{1}\right) \cdot(x b)\left(i G_{1}\right) \\
& =x\left(i F_{1} \cdot{ }^{b}\left(i G_{1}\right)\right)=x\left(i F_{1} \cdot{ }^{i F_{2}}\left(i G_{1}\right)\right)=x\left(i(F G)_{1}\right) .
\end{aligned}
$$

Hence we see that $((\alpha, b)(\beta, c)) \Phi=F G$, and the proof is complete.

LEMMA 2.7. Let $A_{i}(i \in I)$ and $B$ be regular semigroups such that either $A_{i} \in$ $\mathscr{C} \mathscr{S}$ for every $i \in I$ or $B \in \mathscr{C} \mathscr{S}$. Define a mapping $\Phi_{r}:\left(\prod_{i \in I} A_{i}\right)$ wrr $B \rightarrow$ $\prod_{i \in I}\left(A_{i}\right.$ wrr $\left.B\right)$ as follows: for every $(\alpha, b) \in\left(\prod_{i \in I} A_{i}\right)$ wrr $B$, let $(\alpha, b) \Phi_{r}$ be $F \in \prod_{i \in I}\left(A_{i}\right.$ wrr $\left.B\right)$, where, for each $i \in I, i F=\left(i F_{1}, i F_{2}\right) \in A_{i}$ wrr $B$ such that $i F_{2}=b$ and $x\left(i F_{1}\right)=i(x \alpha)$ for any $x \in B^{1}$. Then $\Phi_{r}$ is an embedding. 
PROOF. The mapping $\Phi_{r}$ defined here is the restriction to regular elements of the embedding $\Phi$ given in the previous lemma. Since $\Phi$ maps regular elements to regular ones, and an element in the direct product $\prod_{i \in I}\left(A_{i}\right.$ wrr $\left.B\right)$ is regular if and only if each of its components is regular, we see that $\left(\operatorname{Reg}\left(\left(\prod_{i \in I} A_{i}\right)\right.\right.$ wr $\left.\left.B\right)\right) \Phi \subseteq$ $\prod_{i \in I} \operatorname{Reg}\left(A_{i}\right.$ wr $\left.B\right)$. Thus the assertion follows by Lemma 2.6 .

We can summarize the results obtained so far as follows.

PROPOSITION 2.1. Let $\mathscr{X} \subseteq \mathscr{R} \mathscr{S}$ and let $B \in \mathscr{R} \mathscr{S}$ such that either $\mathscr{X} \subseteq \mathscr{C} \mathscr{S}$ or $B \in \mathscr{C} \mathscr{S}$. Let $\mathbf{Q}$ be one of the operators $\mathbf{H}, \mathbf{S}_{r}, \mathbf{P}, \mathbf{D}^{\infty}$ and $\mathbf{E}=\langle\rangle_{e}$. Then, for any $Q \in \mathbf{Q} \mathscr{X}$, we have $Q$ wrr $B \in \mathbf{S}, \mathbf{Q}\{X$ wrr $B: X \in \mathscr{X}\}$ if $\mathbf{Q}=\mathbf{P}$ and $Q$ wrr $B \in \mathbf{Q}\{X$ wrr $B: X \in \mathscr{X}\}$ otherwise.

PROOF. To get the statement for the operators $\mathbf{H}, \mathbf{S}_{\mathbf{r}}$ and $\mathbf{P}$, we apply Lemma 2.3, Lemma 2.5 and Lemma 2.7, respectively. By applying the statements obtained for $\mathbf{H}$ and $\mathbf{S}_{r}$, an induction on $n$ shows that, for every natural number $n$, we have $Q$ wrr $B \in$ $\left(\mathbf{H S}_{r}\right)^{n}\{X$ wrr $B: X \in \mathscr{X}\}$ provided $Q \in\left(\mathbf{H S}_{r}\right)^{n} \mathscr{X}$. Hence we infer the statement for $\mathbf{D}^{\infty}$. Finally, the statements for $\mathbf{D}^{\infty}$ and $\mathbf{P}$ allow us to prove by transfinite induction that, for every ordinal $i$, we have $Q$ wrr $B \in \mathbf{E}_{i}\{X$ wrr $B: X \in \mathscr{X}\}$ provided $Q \in \mathbf{E}_{i} \mathscr{X}$. Thus we deduce the statement for $\mathbf{E}=\langle\rangle_{e}$. -

Now we are ready to prove the main result of the section.

THEOREM 2.1. Let $\mathscr{U}, \mathscr{V}$ be e-varieties such that $\mathscr{U}$ or $\mathscr{V} \subseteq \mathscr{C} \mathscr{S}$, and let $\mathscr{X} \subseteq \mathscr{U}$ such that $\mathscr{U}=\langle\mathscr{X}\rangle_{e}$. Then $\mathscr{U} *_{r} \mathscr{V}=\langle X \text { wrr } B: X \in \mathscr{X}, B \in \mathscr{V}\rangle_{e}$.

PROOF. By Result 1.1, we have $\mathscr{U} *_{r} \mathscr{V}=\langle A \text { wrr } B: A \in \mathscr{U}, B \in \mathscr{V}\rangle_{e}$. Hence the inclusion $\mathscr{U} *_{r} \mathscr{V} \supseteq\langle X \text { wrr } B: X \in \mathscr{X}, B \in \mathscr{V}\rangle_{e}$ is obvious, and, in order to prove the reverse inclusion, it suffices to show that, for any $A \in \mathscr{U}$ and $B \in \mathscr{V}$, we have $A$ wrr $B \in\langle X \text { wrr } B: X \in \mathscr{X}, B \in \mathscr{V}\rangle_{e}$. However, this follows immediately from Proposition 2.1.

This theorem has two important corollaries. The first one is applied in Section 4 when proving associativity of the regular semidirect product under certain conditions.

COROLLARY 2.1. Let $\mathscr{U}, \mathscr{V}, \mathscr{W}$ be e-varieties such that at least two of them are contained in $\mathscr{C} \mathscr{S}$. Then $\left(\mathscr{U} *_{r} \mathscr{V}\right) *_{r} \mathscr{W}$ is defined, and it is generated by

$$
\mathscr{Y}=\{(A \text { wrr } B) \text { wrr } C: A \in \mathscr{U}, B \in \mathscr{V}, C \in \mathscr{W}\} .
$$

PROOF. The first assertion is obvious by Result 1.5 and by definition. The second one follows from Theorem 2.1, since $\mathscr{U} *_{r} \mathscr{V}=\langle A \text { wrr } B: A \in \mathscr{U}, B \in \mathscr{V}\rangle_{e}$ by Result 1.1. 
The second corollary is the right distributivity of the regular semidirect product with respect to the join operation.

COROLLARY 2.2. Let $\mathscr{U}_{i}(i \in I)$ and $\mathscr{V}$ be e-varieties such that either $\mathscr{U}_{i} \subseteq \mathscr{C} \mathscr{S}$ for each $i \in I$ or $\mathscr{V} \subseteq \mathscr{C} \mathscr{S}$. Then we have $\left(\bigvee_{i \in I} \mathscr{U}_{i}\right) *_{r} \mathscr{V}=\bigvee_{i \in I}\left(\mathscr{U}_{i} *_{r} \mathscr{V}\right)$.

PROOF. Notice that $\bigvee_{i \in I} \mathscr{U}_{i}=\left\langle\bigcup_{i \in I} \mathscr{U}_{i}\right\rangle_{e}$, and apply Theorem 2.1 to see that

$$
\left(\bigvee_{i \in I} \mathscr{U}_{i}\right) *_{r} \mathscr{V}=\left\langle X \text { wrr } B: X \in \bigcup_{i \in I} \mathscr{U}_{i}, B \in \mathscr{V}\right\rangle_{e} \subseteq\left\langle\bigcup_{i \in I}\left(\mathscr{U}_{i} *_{r} \mathscr{V}\right)\right\rangle_{e}=\bigvee_{i \in I}\left(\mathscr{U}_{i} *_{r} \mathscr{V}\right)
$$

The reverse inclusion is obvious.

\section{Generating sets of $\mathscr{U} *_{r} \mathscr{V}$ via those of $\mathscr{V}$}

The topic of this section is similar to the topic of Section 2. It is natural to try to follow the line developed in the previous section. However, the analogue of Lemma 2.6 (or, equivalently, that of Lemma 2.7) does not hold even for groups (see $[14$, pp. 39-40]). Thus the analogues of Theorem 2.1 and Corollary 2.1 are proved only under certain restrictions.

For our later convenience, first we introduce a generalization of the wreath product and regular wreath product constructions.

Let $A, B$ and $\hat{B}$ be semigroups such that $B \leq \hat{B}$. Then $S=\{(\alpha, b) \in A$ wr $\hat{B}$ : $b \in B\}$ is a subsemigroup in $A$ wr $\hat{B}$, and it is a semidirect product of the direct power $A^{\hat{B}^{\prime}}$ of $A$ by $B$. The action is, however, not necessarily left unitary if $B$ has an identity. We denote this $S$ by $A$ wr $B[\hat{B}]$. In particular, if $A$ and $B$ are regular semigroups and $A$ or $B \in \mathscr{C} \mathscr{S}$, then $\operatorname{Reg}(A$ wr $B[\hat{B}])$ is a regular subsemigroup in $A$ wr $B[\hat{B}]$, and we denote it by $A$ wrr $B[\hat{B}]$.

Now we verify the analogues of Lemma 2.2 through Lemma 2.5 .

LEMMA 3.1. Let $A, B, B^{\prime}$ be semigroups such that $B^{\prime} \leq B$. Then $A$ wr $B^{\prime}[B]$ is a subsemigroup in $A$ wr $B$, and the mapping $\Phi: A$ wr $B^{\prime}[B] \rightarrow A$ wr $B^{\prime}$ defined by $(\alpha, b) \Phi=\left(\left.\alpha\right|_{\left(B^{\prime}\right)^{1}}, b\right)$ is a surjective homomorphism.

ProOF. Obvious.

LEMMA 3.2. Let $A, B, B^{\prime}$ be regular semigroups such that $A$ or $B \in \mathscr{C} \mathscr{S}$ and $B^{\prime} \leq B$. Then $A$ wrr $B^{\prime}[B]$ is a regular subsemigroup in $A$ wrr $B$, and the mapping $\Phi_{r}: A$ wrr $B^{\prime}[B] \rightarrow A$ wrr $B^{\prime}$ defined by $(\alpha, b) \Phi_{r}=\left(\left.\alpha\right|_{\left(B^{\prime}\right)^{\prime}}, b\right)$ is a surjective homomorphism. 
PROOF. The first assertion is clear. The mapping $\Phi_{r}$ is a restriction to regular elements of the homomorphism $\Phi$ defined in the previous lemma. Therefore, all we have to show is that if $(\beta, b) \in \operatorname{Reg}\left(A\right.$ wr $\left.B^{\prime}\right)$, then there exists $\alpha \in A^{B^{\prime}}$ such that $\left.\alpha\right|_{\left(B^{\prime}\right)^{1}}=\beta$ and $(\alpha, b) \in \operatorname{Reg}\left(A\right.$ wr $\left.B^{\prime}[B]\right)$.

Since $(\beta, b) \in \operatorname{Reg}\left(A\right.$ wr $\left.B^{\prime}\right)$, Lemma 2.1 ensures that there exists $b^{\prime} \in V(b)$ such that ${ }^{b b^{\prime}} \beta \geq \mathscr{L} \beta$ in $A^{\left(B^{\prime}\right)^{1}}$, that is, $\left(y b b^{\prime}\right) \beta \geq \mathscr{L} y \beta$ in $A$ for every $y \in\left(B^{\prime}\right)^{1}$. Let us choose and fix an element $r$ in $A$, and define $\alpha \in A^{B^{1}}$ in the following manner: for every $x \in B^{1}$, put

$$
x \alpha= \begin{cases}x \beta & \text { if } x \in\left(B^{\prime}\right)^{1} ; \\ \left(x b b^{\prime}\right) \beta & \text { if } x \notin\left(B^{\prime}\right)^{1} \text { and } x b b^{\prime} \in B^{\prime} ; \\ r & \text { if } x b b^{\prime} \notin B^{\prime}\end{cases}
$$

Obviously, we have $\left.\alpha\right|_{\left(B^{\prime}\right)}=\beta$. By the property of $\beta$ mentioned above, we have $\left(x b b^{\prime}\right) \alpha \geq \mathscr{L} x \alpha$ for every $x \in\left(B^{\prime}\right)^{1}$. Furthermore, if $x \notin\left(B^{\prime}\right)^{1}$, then the equality $\left(x b b^{\prime}\right) \alpha=x \alpha$ follows immediately from the definition. Thus ${ }^{b b^{\prime}} \alpha \geq \mathscr{L} \alpha$ in $A^{B^{\prime}}$, and so $(\alpha, b) \in \operatorname{Reg}\left(A\right.$ wr $\left.B^{\prime}[B]\right)$. The proof is complete.

REMARK 3.1. Notice that this proof does not utilize the fact that $B$ is regular. It suffices to assume that $B^{\prime}$ is regular and $A$ or $B^{\prime} \in \mathscr{C} \mathscr{S}$. Thus the following more general statement also holds. Let $A, B, \hat{B}$ be semigroups such that $A, B$ are regular, $A$ or $B \in \mathscr{C} \mathscr{S}$ and $B \leq \hat{B}$. Then the mapping $\Upsilon: A$ wrr $B[\hat{B}] \rightarrow A$ wrr $B$ defined by $(\alpha, b) \Upsilon=\left(\left.\alpha\right|_{B^{1}}, b\right)$ is a surjective homomorphism.

LEMMA 3.3. Let $A, B, \bar{B}$ be semigroups and $\phi: B \rightarrow \bar{B}$ a surjective homomorphism. Then

$$
P=\left\{(\alpha, b) \in A \text { wr } B: \operatorname{ker} \phi^{1} \subseteq \operatorname{ker} \alpha\right\}
$$

is a subsemigroup in $A$ wr $B$, and the mapping $\Phi: P \rightarrow A$ wr $\bar{B}$ defined by $(\alpha, b) \Phi=$ $\left(\alpha_{\phi^{1}}, b \phi\right)$-for the definition of $\alpha_{\phi^{1}}$ see Section 1-is a surjective homomorphism.

Proof. Let $\alpha, \beta \in A^{B^{1}}$ with $\operatorname{ker} \phi^{1} \subseteq \operatorname{ker} \alpha, \operatorname{ker} \beta$, and let $b \in B^{1}$. Then, for every $x, y \in B^{1}$ with $x \phi^{1}=y \phi^{1}$, we have $(x b) \phi^{1}=(y b) \phi^{1}$ since $\phi^{1}$ is a homomorphism, and so $(x b, y b) \in \operatorname{ker} \phi^{1}$. This implies $(x b, y b) \in \operatorname{ker} \beta$ whence $x\left({ }^{b} \beta\right)=(x b) \beta=$ $(y b) \beta=y\left({ }^{b} \beta\right)$ follows. Thus we have shown that $\operatorname{ker} \phi^{1} \subseteq \operatorname{ker}^{b} \beta$. Furthermore, we also have $x\left(\alpha \cdot{ }^{b} \beta\right)=x \alpha \cdot(x b) \beta=y \alpha \cdot(y b) \beta=y\left(\alpha \cdot{ }^{b} \beta\right)$, whence $\operatorname{ker} \phi^{1} \subseteq \operatorname{ker}\left(\alpha \cdot{ }^{b} \beta\right)$ can be deduced. Therefore, $(\alpha, b),(\beta, c) \in P$ implies $(\alpha, b)(\beta, c)=\left(\alpha \cdot{ }^{b} \beta, b c\right) \in P$, and so $P$ is, indeed, a subsemigroup in $A$ wr $B$.

If $(\beta, \bar{b}) \in A$ wr $\bar{B}$, then $\left(\phi^{1} \circ \beta, b\right) \in P$ for every $b \in B$ with $b \phi=\bar{b}$, and, clearly, $\left(\phi^{1} \circ \beta, b\right) \Phi=(\beta, \bar{b})$. Thus $\Phi$ is surjective. Now let $(\alpha, b),(\beta, c) \in P$. Then

$$
((\alpha, b)(\beta, c)) \Phi=\left(\alpha \cdot{ }^{b} \beta, b c\right) \Phi=\left(\left(\alpha \cdot{ }^{b} \beta\right)_{\phi^{1}},(b c) \phi\right)
$$


and

$$
(\alpha, b) \Phi(\beta, c) \Phi=\left(\alpha_{\phi^{1}}, b \phi\right)\left(\beta_{\phi^{1}}, c \phi\right)=\left(\alpha_{\phi^{1}} \cdot{ }^{b \phi} \beta_{\phi^{1}}, b \phi \cdot c \phi\right) .
$$

In order to verify that these elements are equal, it suffices to check the equality of the first components. Consider any $x \in B^{1}$, then

$$
\begin{aligned}
\left(x \phi^{1}\right)\left(\alpha_{\phi^{1}} \cdot{ }^{b \phi} \beta_{\phi^{1}}\right) & =\left(x \phi^{1}\right) \alpha_{\phi^{1}} \cdot\left(x \phi^{1}\right)\left({ }^{b \phi} \beta_{\phi^{1}}\right)=x\left(\phi^{1} \circ \alpha_{\phi^{1}}\right) \cdot\left(x \phi^{1} \cdot b \phi\right) \beta_{\phi^{1}} \\
& =x\left(\phi^{1} \circ \alpha_{\phi^{1}}\right) \cdot\left((x b) \phi^{1}\right) \beta_{\phi^{1}}=x\left(\phi^{1} \circ \alpha_{\phi^{1}}\right) \cdot(x b)\left(\phi^{1} \circ \beta_{\phi^{1}}\right) \\
& =x \alpha \cdot(x b) \beta=x\left(\alpha \cdot{ }^{b} \beta\right) .
\end{aligned}
$$

Since $\left(\alpha \cdot{ }^{b} \beta\right)_{\phi^{1}}$ is the unique mapping such that $\alpha \cdot{ }^{b} \beta=\phi^{1} \circ\left(\alpha \cdot{ }^{b} \beta\right)_{\phi^{1}}$, we infer that $\left(\alpha \cdot{ }^{b} \beta\right)_{\phi^{\prime}}=\alpha_{\phi^{\prime}} \cdot{ }^{b \phi} \beta_{\phi^{\prime}}$. This shows that $\Phi$ is a homomorphism.

LEMMA 3.4. Let $A, B, \bar{B}$ be regular semigroups such that $A$ or $B \in \mathscr{C} \mathscr{S}$, and let $\phi: B \rightarrow \bar{B}$ be a surjective homomorphism. Then

$$
P_{r}=\left\{(\alpha, b) \in A \text { wrr } B: \operatorname{ker} \phi^{1} \subseteq \operatorname{ker} \alpha\right\}
$$

is a regular subsemigroup in $A$ wrr $B$, and the mapping $\Phi_{r}: P_{r} \rightarrow A$ wrr $\bar{B}$ defined by $(\alpha, b) \Phi_{r}=\left(\alpha_{\phi^{1}}, b \phi\right)$ is a surjective homomorphism.

Proof. Clearly, $P_{r}=P \cap(A$ wrr $B)$, where $P$ is the subsemigroup in $A$ wr $B$ introduced in the previous lemma. Thèrefore, $P_{r}$ is a subsemigroup in $A$ wrr $B$. We intend to prove that $P_{r}$ is regular. Let $(\alpha, b) \in P_{r}$, then it is a regular element in $A$ wr $B$, and so, by Lemma 2.1, there exists $b^{\prime} \in V(b)$ such that ${ }^{b b^{\prime}} \alpha \geq \mathscr{L} \alpha$ in $A^{B^{\prime}}$. Let us choose and fix an inverse $a^{\prime}$ of $a$ for each $a \in A$. Define $\xi \in A^{B^{1}}$ in the way that $x \xi=(x \alpha)^{\prime}$ for every $x \in B^{1}$. Then, clearly, we have $\xi \in V(\alpha)$, and Lemma 2.1 ensures that $\left({ }^{b} \xi, b^{\prime}\right) \in V((\alpha, b))$. However, $\operatorname{ker} \phi^{1} \subseteq \operatorname{ker} \alpha$, which implies by the definition of $\xi$ that also $\operatorname{ker} \phi^{1} \subseteq \operatorname{ker} \xi$ and $\operatorname{ker} \phi^{1} \subseteq \operatorname{ker}^{b^{\prime}} \xi$. Thus $\left(b^{\prime} \xi, b^{\prime}\right) \in P_{r}$, showing that $(\alpha, b)$ is regular also in $P_{r}$. Consequently, we have $P_{r}=\operatorname{Reg}(P) \leq_{r} A$ wrr $B$.

The mapping defined in the lemma is the restriction to $P_{r}$ of the homomorphism given in the previous lemma. Therefore, in order to prove the second statement, it suffices to show that if $(\beta, \bar{b}) \in \operatorname{Reg}(A \mathrm{wr} \bar{B})$, then there exists $(\alpha, b) \in P_{r}$ such that $(\alpha, b) \Phi=(\beta, \bar{b})$. Since $(\beta, \bar{b}) \in \operatorname{Reg}(A$ wr $\bar{B})$, by Lemma 2.1 , there exists $\bar{b}^{\prime} \in V(\bar{b})$ such that ${ }^{\bar{b} \bar{b}^{\prime}} \beta \geq \mathscr{L} \beta$ in $A^{\bar{B}^{\prime}}$, that is, $\left(y \bar{b} \bar{b}^{\prime}\right) \beta \geq \mathscr{L} y \beta$ in $A$ for every $y \in \bar{B}^{1}$. Since $B$ is regular, there exists a pair $b, b^{\prime}$ of mutual inverse elements in $B$ such that $b \phi=\vec{b}$ and $b^{\prime} \phi=\bar{b}^{\prime}$. Define $\alpha=\phi^{1} \circ \beta$. Then $(\alpha, b) \in P$ and $(\alpha, b) \Phi=(\beta, \bar{b})$ immediately follow. Moreover, for every $x \in B^{1}$, we have

$$
\begin{aligned}
\left(x b b^{\prime}\right) \alpha & =\left(x b b^{\prime}\right)\left(\phi^{1} \circ \beta\right)=\left(\left(x b b^{\prime}\right) \phi^{1}\right) \beta \\
& =\left(x \phi^{1} \cdot \bar{b} \bar{b}^{\prime}\right) \beta \geq \mathscr{L}\left(x \phi^{1}\right) \beta=x\left(\phi^{1} \circ \beta\right)=x \alpha,
\end{aligned}
$$


and so ${ }^{b b^{\prime}} \alpha \geq \mathscr{L} \alpha$ in $A^{B^{1}}$. This implies, by Lemma 2.1 , that $(\alpha, b) \in A$ wrr $B$. Thus $(\alpha, b) \in P_{r}$ which completes the proof.

By making use of Lemma 3.2 and Lemma 3.4 instead of Lemma 2.3 and Lemma 2.5, respectively, an argument similar to that in the proof of Proposition 2.1 verifies the following analogue of Proposition 2.1.

Proposition 3.1. Let $A \in \mathscr{R} \mathscr{S}$ and let $\mathscr{X} \subseteq \mathscr{R} \mathscr{S}$ such that either $A \in \mathscr{C} \mathscr{S}$ or $\mathscr{X} \subseteq \mathscr{C} \mathscr{S}$. Then, for any $Q \in \mathbf{S}_{r} \mathscr{X} \cup \mathbf{H} \mathscr{X}$, we have $A$ wrr $Q \in \mathbf{H S}_{r}[A$ wrr $X$ : $X \in \mathscr{X}\}$, and for any $Q \in \mathrm{D}^{\infty} \mathscr{X}$, we have $A$ wrr $Q \in \mathrm{D}^{\infty}\{A$ wrr $X: X \in \mathscr{X}\}$.

This proposition implies the following analogue of Theorem 2.1 .

THEOREM 3.1. Let $\mathscr{U}, \mathscr{V}$ be e-varieties such that $\mathscr{U}$ or $\mathscr{V} \subseteq \mathscr{C} \mathscr{S}$, and let $\mathscr{X} \subseteq \mathscr{V}$ such that $\mathscr{V}=\mathbf{D}^{\infty} \mathscr{X}$. Then $\mathscr{U} *_{r} \mathscr{V}=\langle A \text { wrr } X: A \in \mathscr{U}, X \in \mathscr{X}\rangle_{e}$.

The condition that $\mathscr{V}=\mathrm{D}^{\infty} \mathscr{X}$ seems to be rather strong in general. However, if $\mathscr{X} \subseteq L \mathscr{I}$ or $\mathscr{E} \mathscr{S}$, then $\mathbf{D}^{\infty} \mathscr{X}=\mathbf{H S}, \mathscr{X}$, and so $\mathscr{V}=\mathbf{D}^{\infty} \mathscr{X}$ is satisfied if and only if $\mathscr{V}=\langle\mathscr{X}\rangle_{e}$ and $\mathbf{P} \mathscr{X} \subseteq \mathbf{H S}_{r} \mathscr{X}$. Thus we obtain the following corollary.

COROLLARY 3.1. Let $\mathscr{U}, \mathscr{V}$ be e-varieties'such that either $\mathscr{V} \subseteq \mathscr{C} \mathscr{S}$ or both $\mathscr{U} \subseteq \mathscr{C} \mathscr{S}$ and $\mathscr{V} \subseteq L \mathscr{I}$ or $\mathscr{E} \mathscr{S}$. Let $\mathscr{X} \subseteq \mathscr{V}$ such that $\mathscr{V}=\langle\mathscr{X}\rangle_{e}$ and $\mathbf{P} \mathscr{X} \subseteq \mathbf{H S}_{r} \mathscr{X}$. Then $\mathscr{U} *_{r} \mathscr{V}=\langle A \text { wrr } X: A \in \mathscr{U}, X \in \mathscr{X}\rangle_{e}$.

Let us notice that the extra condition on the generating set involved in this corollary is satisfied by the generating set $\mathscr{X}$ of $\mathscr{U} *_{r} \mathscr{V}$ presented in Result 1.1. We prove somewhat more. Denote the generating set given in the definition of $\mathscr{U} *_{r} \mathscr{V}$ by $\mathscr{X}^{*}$. We have seen that $\mathscr{X} \subseteq \mathscr{X}^{*} \subseteq \mathbf{S}_{r} \mathscr{X}$. First we show that $\mathbf{P} \mathscr{X}^{*} \subseteq \mathscr{X}^{*}$. If $A_{i} \in \mathscr{U}, B_{i} \in \mathscr{V}$ and $B_{i}$ acts on $A_{i}$ for every $i \in I$, then $\prod_{i \in I} B_{i}$ acts on $\prod_{i \in I} A_{i}$ componentwise. Note that $\prod_{i \in I} B_{i}$ has an identity if and only if each $B_{i}(i \in I)$ has an identity, and if this is the case, then the action of $\prod_{i \in I} B_{i}$ on $\prod_{i \in I} A_{i}$ is left unitary if and only if the action of $B_{i}$ on $A_{i}$ is left unitary for all $i \in I$. Thus $\mathbf{P} \mathscr{X}^{*} \subseteq \mathscr{X}^{*}$ follows. This implies $\mathbf{P} \mathscr{X} \subseteq \mathbf{P} \mathscr{X}^{*} \subseteq \mathscr{X}^{*} \subseteq \mathbf{S}_{r} \mathscr{X}$.

Now, by applying Corollary 3.1 instead of Theorem 2.1, the argument in the proof of Corollary 2.1 shows that $\mathscr{U} *_{r}\left(\mathscr{V} *_{r} \mathscr{W}\right)=\langle A$ wrr $(B$ wrr $C): A \in \mathscr{U}, B \in \mathscr{V}, C \in$ $\mathscr{W}\rangle_{e}$ provided at least two of $\mathscr{U}, \mathscr{V}, \mathscr{W}$ are in $\mathscr{C} \mathscr{S}$ and $\mathscr{V} *_{r} \mathscr{W} \subseteq L \mathscr{I}$ or $\mathscr{E} \mathscr{S}$. Thus we deduce the following corollary.

COROLLARY 3.2. Let $\mathscr{U}, \mathscr{V}, \mathscr{W}$ be e-varieties such that at least two of them are contained in $\mathscr{C} \mathscr{S}$. Then $\mathscr{U} *_{r}\left(\mathscr{V} *_{r} \mathscr{W}\right)$ is defined, and

$$
\mathscr{Z}=\{A \operatorname{wrr}(B \operatorname{wrr} C): A \in \mathscr{U}, B \in \mathscr{V}, C \in \mathscr{W}\}
$$


is a subclass in $\mathscr{U} *_{r}\left(\mathscr{V} *_{r} \mathscr{W}\right)$. If, moreover, $\mathscr{V} *_{r} \mathscr{W} \subseteq L \mathscr{I}$ or $\mathscr{E} \mathscr{S}$ (see Result 1.2), then $\mathscr{U} *_{r}\left(\mathscr{V} *_{r} \mathscr{W}\right)$ is generated by $\mathscr{Z}$.

\section{Associativity of the regular semidirect product}

The aim of this section is to prove by applying the tools developed in the former sections that $\left(\mathscr{U} *_{r} \mathscr{V}\right) *_{r} \mathscr{W}=\mathscr{U} *_{r}\left(\mathscr{V} *_{r} \mathscr{W}\right)$ holds for the e-varieties $\mathscr{U}, \mathscr{V}, \mathscr{W}$ if either $\mathscr{U} \subseteq \mathscr{C} \mathscr{S}$ and $\mathscr{V} *_{r} \mathscr{W} \subseteq L \mathscr{I}$ or $\mathscr{E} \mathscr{S}$, or one of $\mathscr{V}, \mathscr{W}$ is contained in $\mathscr{G}$ and the other one in $\mathscr{C} \mathscr{S}$, or $\mathscr{V}=\mathscr{L} \mathscr{Z}$ and $\mathscr{W} \subseteq \mathscr{C} \mathscr{S}$. First we determine under what conditions a regular wreath product has an identity element. Then we introduce a construction originating in the wreath product of transformation semigroups (see $[4,13]$ ), and obtain a new generating set $\mathscr{Z}^{\star}$ for the e-varieties considered in Corollary 3.2. Finally, we compare $\mathscr{Y}$ and $\mathscr{Z}^{*}$, thus providing the sufficient conditions for associativity mentioned above.

First we show that a wreath product $A$ wr $B$ [a regular wreath product $A$ wrr $B$ ] has an identity if and only if both $A$ and $B$ have identities.

LEMMA 4.1. Let $A$ and $B$ be any semigroups. Then $A$ wr $B$ has an identity if and only if both $A$ and $B$ have identities. If this is the case, then the identity of $A$ wr $B$ is $\left(\epsilon_{A}, 1_{B}\right)$, where $\epsilon_{A} \in A^{B}$ is such that $x \epsilon_{A}=1_{A}$ for every $x \in B$.

Proof. Suppose that $(\alpha, b) \in A$ wr $B$ is an identity. Since the second projection of $A$ wr $B$ to $B$ is a surjective homomorphism, we infer that $b=1_{B} \in B$. Moreover, for every $\xi \in A^{B}$, we have $\left(\alpha, 1_{B}\right)\left(\xi, 1_{B}\right)=\left(\xi, 1_{B}\right)\left(\alpha, 1_{B}\right)=\left(\xi, 1_{B}\right)$, whence it follows that $\alpha=1_{A^{B}} \in A^{B}$. Since the direct power $A^{B}$ of $A$ has an identity if and only if $A$ has an identity, and in this case, $1_{A^{B}}=\epsilon_{A}$, we obtain that $\alpha=\epsilon_{A}$. Conversely, it is straightforward to check that if $1_{A} \in A$ and $1_{B} \in B$, then $\left(\epsilon_{A}, 1_{B}\right)$ is an identity in $A$ wr $B$.

Now we investigate the identity element in the regular wreath product.

LEMMA 4.2. Let $A$ and $B$ be regular semigroups such that $A$ or $B \in \mathscr{C} \mathscr{S}$. Then $A$ wrr $B$ has an identity if and only if both $A$ and $B$ have identities. If this is the case, then the identity of $A$ wrr $B$ is $\left(\epsilon_{A}, 1_{B}\right)$, where $\epsilon_{A} \in A^{B}$ is such that $x \epsilon_{A}=1_{A}$ for every $x \in B$.

PROOF. The argument in the previous lemma applies if we notice that the second projection of $A$ wrr $B$ to $B$ is surjective, and $\left(\xi, 1_{B}\right) \in A$ wrr $B$ for every $\xi \in A^{B}$ provided $1_{B} \in B$. The latter property is clear. By Lemma 2.1 , we have $\left({ }^{(} \xi, y\right) \in$ $A$ wrr $B$ for every $\xi \in A^{B}$ and $y \in B$. Hence the second projection of $A$ wrr $B$ to $B$ is, indeed, surjective. 
REMARK 4.1. It is easy to see that the above proofs apply not only for the wreath products $A$ wr $B$ and $A$ wrr $B$ but also for any semidirect products $A * B$ and $A *_{r} B$, respectively, where the action of $B$ on $A$ has the following two properties: (i) the action is left unitary provided $B$ has an identity, and (ii) $B$ acts on $A$ by monoid endomorphisms (that is, ${ }^{b} 1_{A}=1_{A}$ for every $b \in B$ ) if $A$ has an identity.

Lemma 4.1 and Lemma 4.2 allow us to identify $1_{A \text { wr } B}$ and $1_{A \text { wr } B}$ with $\left(\epsilon_{A}, 1_{B}\right)$. More precisely, if $1_{A} \in A$ and $1_{B} \in B$, then we proved $1_{A \text { шг } B}=1_{A \text { шп } B}=\left(\epsilon_{A}, 1_{B}\right)$. In the opposite case, $1_{A \text { wr } B} \notin A$ wr $B$ and $1_{A \text { wr } B} \notin A$ wrr $B$. Then we can adjoin identities to $A$ and $B$, and we can choose both $1_{A \text { wr } B}$ and $1_{A \text { wrr } B}$ to be $\left(\epsilon_{A}, 1_{B}\right)$, where $\epsilon_{A} \in\left(A^{1}\right)^{B^{1}}$ such that $x \epsilon_{A}=1_{A}$ for every $x \in B^{1}$. This is convenient for us because in this way $(A \mathrm{wr} B)^{1}$ and $(A \text { wrr } B)^{1}$ are subsemigroups in $A^{1}$ wr $B^{1}$.

The following construction which comes from the notion of the wreath product of transformation semigroups plays a crucial role in this section.

Let $A, B, C$ be semigroups. The wreath product $B$ wr $C$ acts naturally on the direct power $A^{B^{1} \times C^{1}}$ of $A$ as follows. For every $(\beta, c) \in B$ wr $C$ and $\alpha \in A^{B^{1} \times C^{1}}$, define ${ }^{(\beta, c)} \alpha$ to be the mapping $B^{1} \times C^{1} \rightarrow A$ given by $(x, y)\left({ }^{(\beta, c)} \alpha\right)=(x \cdot y \beta, y c) \alpha$. It is straightforward to see that this is, indeed, an action. This action defines a semidirect product of $A^{B^{1} \times C^{1}}$ by $B$ wr $C$ which we denote by $A^{B^{1} \times C^{1}} \star(B$ wr $C)$.

Suppose now that $A, B, C$ are regular and at least two of them are in $\mathscr{C} \mathscr{S}$. Then $B$ wrr $C$ is defined, and the restriction of the action of $B$ wr $C$ on $A^{B^{1} \times C^{1}}$ to $B$ wrr $C$ determines a semidirect product of $A^{B^{1} \times C^{1}}$ by $B$ wrr $C$ which we also denote by $A^{B^{1} \times C^{1}} \star(B$ wrr $C)$. Clearly, $A^{B^{1} \times C^{1}} \star(B$ wrr $C)$ is a subsemigroup in $A^{B^{1} \times C^{1}} \star(B$ wr $C)$. Futhermore, $\operatorname{Reg}\left(A^{B^{1} \times C^{1}} \star(B\right.$ wrr $C)$ ) forms a (regular) subsemigroup in $A^{B^{1} \times C^{1}} \star$ $\left(B\right.$ wrr $C$ ) which we denote by $A^{B^{1} \times C^{1}} \star_{r}(B$ wrr $C)$.

Note that the action of $B$ wr $C[B$ wrr $C]$ on $A^{B^{1} \times C^{1}}$ is left unitary if $B$ wr $C[B$ wrr $C]$ has an identity. For, if $B$ wr $C$ [ $B$ wrr $C$ ] has an identity, then, by Lemma 4.1 [4.2], we have $1_{B} \in B, 1_{C} \in C$, and the identity of $B$ wr $C[B$ wrr $C]$ is $\left(\epsilon_{B}, 1_{C}\right)$. Thus, for every $\alpha \in A^{B^{1} \times C^{1}}$ and $(x, y) \in B^{1} \times C^{1}=B \times C$, we have $(x, y)\left({ }^{\left(\epsilon_{B}, I_{C}\right)} \alpha\right)=$ $\left(x \cdot y \epsilon_{B}, y \cdot 1_{C}\right) \alpha=\left(x \cdot 1_{B}, y \cdot 1_{C}\right) \alpha=(x, y) \alpha$.

Now we are ready to present a new generating set for the e-varieties $\mathscr{U} *_{r}\left(\mathscr{V} *_{r} \mathscr{W}\right)$ mentioned in Corollary 3.2.

PROPOSITION 4.1. Let $\mathscr{U}, \mathscr{V}, \mathscr{W}$ be e-varieties such that at least two of them are contained in $\mathscr{C} \mathscr{S}$. Then $\mathscr{U} *_{r}\left(\mathscr{V} *_{r} \mathscr{W}\right)$ is defined, and

$$
\mathscr{Z}^{\star}=\left\{A^{B^{1} \times C^{1}} \star_{r}(B \text { wrr } C): A \in \mathscr{U}, B \in \mathscr{V}, C \in \mathscr{W}\right\}
$$

is a subclass in $\mathscr{U} *_{r}\left(\mathscr{V} *_{r} \mathscr{W}\right)$. If, moreover, $\mathscr{V} *_{r} \mathscr{W} \subseteq L \mathscr{I}$ or $\mathscr{E} \mathscr{S}$ (see Result 1.2), then $\mathscr{U} *_{r}\left(\mathscr{V} *_{r} \mathscr{W}\right)$ is generated by $\mathscr{Z}^{\star}$. 
Proof. The first statement is clear by definition. Therefore, by Corollary 3.2, it suffices to verify that $A$ wrr $(B$ wrr $C) \in \mathbf{H S}_{r} \mathscr{Z}^{*}$ for every $A \in \mathscr{U}, B \in \mathscr{V}, C \in \mathscr{W}$. The statement in Remark 3.1 implies that $A$ wrr $(B$ wrr $C)$ is a homomorphic image of $A$ wrr $(B$ wrr $C)\left[B^{1}\right.$ wr $\left.C^{1}\right]$. Now we prove that the latter semigroup is embeddable into $\left(A^{B^{1} w r} C^{1}\right)^{\left(B^{C^{1}}\right)^{1} \times C^{1}} \star_{r}\left(B^{C^{1}}\right.$ wrr $\left.C\right)$.

Let us define a mapping

$$
\Phi: A \text { wr }(B \text { wrr } C)\left[B^{1} \text { wr } C^{1}\right] \rightarrow\left(A^{B^{1} \text { wr } C^{1}}\right)^{\left(B^{c^{1}}\right)^{1} \times C^{1}} \star\left(B^{C^{1}} \text { wrr } C\right)
$$

in the way that $(\alpha,(\beta, c)) \Phi=(\widetilde{\alpha},(\widetilde{\beta}, c))$, where $\widetilde{\beta}: C^{1} \rightarrow B^{C^{1}}$ is given by $x \widetilde{\beta}={ }^{x} \beta$ for every $\beta \in B^{C^{1}}$, and $\widetilde{\alpha}:\left(B^{C^{1}}\right)^{1} \times C^{1} \rightarrow A^{B^{1} \text { wr } C^{1}}$ is defined by $(\xi, x) \widetilde{\alpha}={ }^{(\xi, x)} \alpha$ for every $\xi \in\left(B^{C^{1}}\right)^{1}$ and $x \in C^{1}$. Since $B^{C^{1}} \leq\left(B^{1}\right)^{C^{1}}$, by our convention, we have $1_{B^{\mathrm{C}^{1}}}=\epsilon_{B}$, the identity in $\left(B^{1}\right)^{C^{1}}$. Therefore, $\xi$ can be considered as an element in $\left(B^{1}\right)^{C^{1}}$, and so $(\xi, x) \in B^{1}$ wr $C^{1}$. Since $(\widetilde{\beta}, c)$ is the image of $(\beta, c)$ via the natural embedding of $B$ wrr $C$, considered as a regular semidirect product $B^{C^{1}} *_{r} C$, into the regular wreath product $B^{C^{1}}$ wrr $C$, we see that $(\widetilde{\beta}, c)$ is, indeed, in $B^{C^{1}}$ wrr $C$. Let $(\alpha,(\beta, c)),(\bar{\alpha},(\bar{\beta}, \bar{c})) \in A \operatorname{wr}(B \operatorname{wrr} C)\left[B^{1} \operatorname{wr} C^{1}\right]$. If $(\alpha,(\beta, c)) \Phi=(\bar{\alpha},(\bar{\beta}, \bar{c})) \Phi$, then $(\beta, c)=(\bar{\beta}, \bar{c})$ follows. The equality $\widetilde{\alpha}=\widetilde{\bar{\alpha}}$ implies $\alpha=\bar{\alpha}$ since $\alpha={ }^{\left({ }_{B}, 1_{c}\right)} \alpha=$ $\left(\epsilon_{B}, 1_{C}\right) \widetilde{\alpha}=\left(\epsilon_{B}, 1_{C}\right) \widetilde{\bar{\alpha}}={ }^{\left(\epsilon_{B}, 1_{c}\right.} \bar{\alpha}=\bar{\alpha}$. Thus we obtain that $\Phi$ is injective.

To see that $\Phi$ is a homomorphism, it suffices to prove the equality of the first components in $((\alpha,(\beta, c)) \cdot(\bar{\alpha},(\bar{\beta}, \bar{c}))) \Phi$ and $(\alpha,(\beta, c)) \Phi \cdot(\bar{\alpha},(\bar{\beta}, \bar{c})) \Phi$, that is, the equality $(\widetilde{\alpha \cdot(\beta, c)} \bar{\alpha})=\widetilde{\alpha} \cdot(\tilde{\beta}, c) \widetilde{\bar{\alpha}}$. For, the observation that $\Phi$ on the second component is a natural embedding ensures the equality required in the second component. For every $(\xi, x) \in\left(B^{C^{1}}\right)^{1} \times C^{1} \subseteq B^{1}$ wr $C^{1}$, we have

$$
\begin{aligned}
(\xi, x)\left(\tilde{\alpha} \cdot{ }^{(\tilde{\beta}, c)} \tilde{\bar{\alpha}}\right) & =(\xi, x) \tilde{\alpha} \cdot(\xi, x)((\widetilde{\beta}, c) \tilde{\bar{\alpha}})=(\xi, x) \tilde{\alpha} \cdot(\xi \cdot x \widetilde{\beta}, x c) \widetilde{\bar{\alpha}} \\
& =(\xi, x) \tilde{\alpha} \cdot\left(\xi \cdot{ }^{x} \beta, x c\right) \widetilde{\bar{\alpha}}=(\xi, x) \tilde{\alpha} \cdot((\xi, x)(\beta, c)) \widetilde{\bar{\alpha}} \\
& ={ }^{(\xi, x)} \alpha \cdot(\xi, x)(\beta, c) \bar{\alpha}={ }^{(\xi, x)}\left(\alpha \cdot{ }^{(\beta, c)} \bar{\alpha}\right)=(\xi, x)(\widetilde{\alpha \cdot(\beta, c) \bar{\alpha}}) .
\end{aligned}
$$

Thus we have shown that $\Phi$ is an embedding. In particular, it assigns regular elements to regular elements. So its restriction to $A$ wrr $(B$ wrr $C)\left[B^{1}\right.$ wr $\left.C^{1}\right]$ is an embedding into $\left(A^{B^{1} \text { wr } C^{1}}\right)^{\left(B^{C^{1}}\right)^{1} \times C^{1}} \star_{r}\left(B^{C^{1}}\right.$ wrr $\left.C\right)$. The latter semigroup obviously belongs to $\mathscr{Z}^{\star}$, and so we proved that $A$ wrr $(B$ wrr $C) \in \mathbf{H S}_{r} \mathscr{Z}^{*}$.

The following lemma is, actually, a consequence of the associativity of the wreath product of transformation semigroups (see [4, 13]). For completeness, we give a proof in our setting.

Lemma 4.3. Let $A, B, C$ be arbitrary semigroups. Define a mapping

$$
\Psi:(A \mathrm{wr} B) \operatorname{wr} C \rightarrow A^{B^{1} \times C^{1}} \star(B \mathrm{wr} C)
$$


as follows. If $(\gamma, c) \in(A \mathrm{wr} B) \mathrm{wr} C$ and $\gamma_{1}, \gamma_{2}$ are the components of the mapping $\gamma: C^{1} \rightarrow A$ wr $B=A^{B^{1}} \times B$, then let $\tilde{\gamma}_{1}: B^{1} \times C^{1} \rightarrow A$ be the mapping defined by $(x, y) \widetilde{\gamma}_{1}=x\left(y \gamma_{1}\right)$, and put $(\gamma, c) \Psi=\left(\widetilde{\gamma}_{1},\left(\gamma_{2}, c\right)\right)$. Then $\Psi$ is an isomorphism.

Proof. First we show that $\Psi$ is injective. Let $(\gamma, c),(\delta, d) \in(A$ wr $B)$ wr $C$ such that $(\gamma, c) \Psi=(\delta, d) \Psi$. Then $\tilde{\gamma}_{1}=\widetilde{\delta}_{1}, \gamma_{2}=\delta_{2}$ and $c=d$. In order to show that $(\gamma, c)=(\delta, d)$, it suffices to verify that $\gamma_{1}=\delta_{1}$. However, the equality $\tilde{\gamma}_{1}=\tilde{\delta}_{1}$ says that, for every $y \in C^{1}, y \gamma_{1}$ and $y \delta_{1}$ are equal mappings in $A^{B^{1}}$ whence $\gamma_{1}=\delta_{1}$ follows.

In order to check surjectivity, let $(\xi,(\beta, c)) \in A^{B^{1} \times C^{1}} \star(B$ wr $C)$. Define $\gamma: C^{1} \rightarrow$ $A$ wr $B=A^{B^{1}} \times B$ by $y \gamma=((-, y) \xi, y \beta)$. Here $(-, y) \xi$ is the mapping $B^{1} \rightarrow A$ such that $x((-, y) \xi)=(x, y) \xi\left(x \in B^{1}\right)$. It is obviuos that $\tilde{\gamma}_{1}=\xi, \gamma_{2}=\beta$, and so $(\gamma, c) \Psi=(\xi,(\beta, c))$.

Finally, we show that $\Psi$ is a homomorphism. Let $(\gamma, c),(\delta, d) \in(A$ wr $B)$ wr $C$. Then, by definition, we see that

$$
((\gamma, c)(\delta, d)) \Psi=\left(\gamma \cdot{ }^{c} \delta, c d\right) \Psi=\left(\widetilde{(\gamma \cdot c \delta)_{1}},\left(\left(\gamma \cdot{ }^{c} \delta\right)_{2}, c d\right)\right) .
$$

Here the following holds in $A$ wr $B$ for every $y \in C^{1}$ :

$$
y\left(\gamma \cdot{ }^{c} \delta\right)=\left(y\left(\gamma \cdot{ }^{c} \delta\right)_{1}, y\left(\gamma \cdot{ }^{c} \delta\right)_{2}\right)
$$

and

$$
\begin{aligned}
y\left(\gamma \cdot{ }^{c} \delta\right) & =y \gamma \cdot(y c) \delta=\left(y \gamma_{1}, y \gamma_{2}\right)\left((y c) \delta_{1},(y c) \delta_{2}\right) \\
& =\left(y \gamma_{1} \cdot{ }^{y r_{2}}(y c) \delta_{1}, y \gamma_{2} \cdot(y c) \delta_{2}\right)=\left(y \gamma_{1} \cdot{ }^{y \gamma_{2}}(y c) \delta_{1}, y\left(\gamma_{2} \cdot{ }^{c} \delta_{2}\right)\right) .
\end{aligned}
$$

Hence we obtain that

$$
y\left(\gamma \cdot{ }^{c} \delta\right)_{1}=y \gamma_{1} \cdot{ }^{y \gamma_{2}}(y c) \delta_{1} \quad \text { for every } y \in C^{1},
$$

and

$$
\left(\gamma \cdot{ }^{c} \delta\right)_{2}=\gamma_{2} \cdot{ }^{c} \delta_{2} .
$$

Furthermore, we have

$$
(\gamma, c) \Psi \cdot(\delta, d) \Psi=\left(\tilde{\gamma}_{1},\left(\gamma_{2}, c\right)\right)\left(\tilde{\delta}_{1},\left(\delta_{2}, d\right)\right)=\left(\tilde{\gamma}_{1} \cdot\left(\gamma_{2}, c \tilde{\delta}_{1},\left(\gamma_{2} \cdot \delta_{2}, c d\right)\right) .\right.
$$

By (2), it is clear that the components of $((\gamma, c)(\delta, d)) \Psi$ and $(\gamma, c) \Psi \cdot(\delta, d) \Psi$ belonging to $B$ wr $C$ coincide. It remains to prove that

$$
\widetilde{(\gamma \cdot c \delta})_{1}=\tilde{\gamma}_{1} \cdot\left(r_{2}, c \tilde{\delta}_{1}\right.
$$

For every $(x, y) \in B^{1} \times C^{1}$, we have

$$
(x, y)\left(\widetilde{\gamma}_{1} \cdot{ }^{\left(\gamma_{2}, c\right.} \tilde{\delta}_{1}\right)=(x, y) \widetilde{\gamma}_{1} \cdot(x, y)\left({ }^{\left(\gamma_{2}, c\right.} \widetilde{\delta}_{1}\right)=(x, y) \tilde{\gamma}_{1} \cdot\left(x \cdot y \gamma_{2}, y c\right) \tilde{\delta}_{1}
$$


and

$$
\begin{aligned}
(x, y) \widetilde{(\gamma \cdot c \delta})_{1} & =x\left(y\left(\gamma \cdot{ }^{c} \delta\right)_{1}\right)=x\left(y \gamma_{1} \cdot y r^{2}(y c) \delta_{1}\right) \quad \text { by }(1) \\
& =x\left(y \gamma_{1}\right) \cdot x\left({ }^{y \gamma_{2}}(y c) \delta_{1}\right)=x\left(y \gamma_{1}\right) \cdot\left(x \cdot y \gamma_{2}\right)\left((y c) \delta_{1}\right) \\
& =(x, y) \widetilde{\gamma_{1}} \cdot\left(x \cdot y \gamma_{2}, y c\right) \widetilde{\delta}_{1},
\end{aligned}
$$

which completes the proof.

Hence we obtain the following consequence for regular wreath products.

LEMMA 4.4. Let $A, B, C$ be regular semigroups such that at least two of them are in $\mathscr{C} \mathscr{S}$. Then both $(A$ wrr $B)$ wrr $C$ and $A^{B^{1} \times C^{1}} \star_{r}(B$ wrr $C)$ are defined, $\operatorname{Reg}((A$ wr $B)$ wr $C)$ constitutes a (regular) subsemigroup in $(A \mathrm{wr} B)$ wr $C$, we have

$$
\text { ( } A \text { wrr } B \text { ) wrr } C \leq_{r} \operatorname{Reg}((A \text { wr } B) \text { wr } C \text { ), }
$$

and the restriction $\Psi_{r}: \operatorname{Reg}((A$ wr $B)$ wr $C) \rightarrow A^{B^{1} \times C^{1}} \star_{r}(B$ wrr $C)$ of the isomorphism $\Psi$ defined in Lemma 4.3 is an isomorphism.

PROOF. It is clear that if $(\xi,(\beta, c)) \in A^{B^{1} \times C^{1}} \star(B$ wr $C)$ is a regular element, then $(\beta, c) \in B$ wr $C$ is necessarily regular. Therefore, $\operatorname{Reg}\left(A^{B^{1} \times C^{1}} \star(B \mathrm{wr} C)\right)=$ $\operatorname{Reg}\left(A^{B^{1} \times C^{1}} \star(B\right.$ wrr $\left.C)\right)=A^{B^{1} \times C^{1}} \star_{r}(B$ wrr $C)$. Since both the isomorphism $\Psi$ and its inverse assign regular elements to regular ones, we infer from Lemma 4.3 that $\operatorname{Reg}((A$ wr $B)$ wr $C)$ is a regular subsemigroup in $(A$ wr $B)$ wr $C$ and $\Psi_{r}$ is an isomorphism. Since $\operatorname{Reg}((A$ wrr $B)$ wr $C) \subseteq \operatorname{Reg}((A$ wr $B)$ wr $C)$, we obtain that $(A$ wrr $B)$ wrr $C \leq_{r} \operatorname{Reg}((A$ wr $B)$ wr $C)$.

An immediate consequence of this lemma is the following result.

LEMMA 4.5. If $\mathscr{U}, \mathscr{V}, \mathscr{W}$ are e-varieties such that at least two of them are contained in $\mathscr{C} \mathscr{S}$, then $\mathscr{Y} \subseteq \mathrm{S}_{r} \mathscr{Z}^{\star}$ holds for the classes defined in Corollary 2.1 and Proposition 4.1, respectively.

This implies the following theorem.

THEOREM 4.1. Let $\mathscr{U}, \mathscr{V}, \mathscr{W}$ be e-varieties such that at least two of them are contained in $\mathscr{C} \mathscr{S}$. Then $\left(\mathscr{U} *_{r} \mathscr{V}\right) *_{r} \mathscr{W} \subseteq \mathscr{U} *_{r}\left(\mathscr{V} *_{r} \mathscr{W}\right)$.

In the rest of this section we look for special cases, where the reverse inclusion also holds. First we find sufficient conditions which ensure that $\mathscr{Y}=\mathscr{Z}^{*}$.

LEMMA 4.6. Let $A, B, C$ be regular semigroups such that $B$ acts on $A$ and $C$ acts on $A * B$, and consider the semidirect product $(A * B) * C$. Suppose that one of the following conditions holds: (i) $A \in \mathscr{C} \mathscr{S}$, (ii) $B \in \mathscr{G}$, (iii) $C \in \mathscr{G}$. Then, for every $((a, b), c) \in \operatorname{Reg}((A * B) * C)$, we have $(a, b) \in \operatorname{Reg}(A * B)$. 
Proof. The statement is obvious in case (ii) since if $B \in \mathscr{G}$, then $A * B=$ $\operatorname{Reg}(A * B)$.

Let $((a, b), c) \in(A * B) * C$ be regular. Then there exists $\left(\left(a^{\prime}, b^{\prime}\right), c^{\prime}\right) \in(A * B) * C$ such that $((a, b), c)\left(\left(a^{\prime}, b^{\prime}\right), c^{\prime}\right)((a, b), c)=((a, b), c)$, that is,

$$
c c^{\prime} c=c \quad \text { and } \quad(a, b) \cdot{ }^{c}\left(a^{\prime}, b^{\prime}\right) \cdot{ }^{c c^{\prime}}(a, b)=(a, b) .
$$

In case (iii), $c^{\prime}$ is the group inverse of $c$ and $c c^{\prime}=1_{c}$. Therefore, the second equality shows that $(a, b) \in \operatorname{Reg}(A * B)$. In case (i), it suffices to observe, by Result 1.3, that ${ }^{c}\left(a^{\prime}, b^{\prime}\right) \cdot{ }^{c c^{\prime}}(a, b)$ is idempotent. This follows since (3) implies ${ }^{c c^{\prime}}(a, b) \cdot{ }^{c}\left(a^{\prime}, b^{\prime}\right)$. ${ }^{c c^{\prime}}(a, b)={ }^{c c^{\prime}}(a, b)$ whence ${ }^{c}\left(a^{\prime}, b^{\prime}\right) \cdot{ }^{c c^{\prime}}(a, b) \in E(A * B)$.

Now we can establish the sufficient conditions mentioned.

LEMMA 4.7. Let $\mathscr{U}, \mathscr{V}, \mathscr{W}$ be e-varieties such that at least two of them are contained in $\mathscr{C} \mathscr{S}$. Assume that, moreover, one of the conditions (i) $\mathscr{U} \subseteq \mathscr{C} \mathscr{S}$, (ii) $\mathscr{V} \subseteq \mathscr{G}$ and (iii) $\mathscr{W} \subseteq \mathscr{G}$ are satisfied. Then $\mathscr{Y}=\mathscr{Z}^{\star}$ holds for the classes defined in Corollary 2.1 and Proposition 4.1, respectively.

Proof. Let $A \in \mathscr{U}, B \in \mathscr{V}$ and $C \in \mathscr{W}$. It is clear that (A wrr $B$ ) wrr $C \subseteq$ $\operatorname{Reg}((A$ wr $B)$ wr $C)$. Conversely, Lemma 4.6 implies that $\operatorname{Reg}((A$ wr $B)$ wr $C) \subseteq$ $\operatorname{Reg}((A$ wrr $B)$ wr $C)$. Since either $C \in \mathscr{C} \mathscr{S}$ or $A$ wrr $B \in \mathscr{C} \mathscr{S}$, we have $\operatorname{Reg}((A$ wrr $B)$ wr $C)=(A$ wrr $B)$ wrr $C$. Thus

$$
\operatorname{Reg}((A \mathrm{wr} B) \operatorname{wr} C)=(A \text { wrr } B) \text { wrr } C,
$$

and so, by Lemma 4.4, $(A$ wrr $B)$ wrr $C$ is isomorphic to $A^{B^{1} \times C^{1}} \star_{r}(B$ wrr $C)$. Hence we deduce that $\mathscr{Y}=\mathscr{Z}^{\star}$.

Another case, where we are able to find a relation between $\mathscr{Y}$ and $\mathscr{Z}^{\star}$ which implies the inclusion reverse to that in Theorem 4.1 is when $\mathscr{V}=\mathscr{L} \mathscr{Z}$ and $\mathscr{W} \subseteq \mathscr{C} \mathscr{S}$ but $\mathscr{W} \nsubseteq \mathscr{G}$. One of the reasons that this case can be handled is that a semidirect product of a left zero semigroup by an arbitrary semigroup is necessarily their direct product.

Let $A, B, C$ be semigroups such that $B \in \mathscr{L} \mathscr{Z}$. Then $B \times C$ has no identity, and so we are allowed to choose $1_{B \times C}=\left(1_{B}, 1_{C}\right)$. Let us identify $B^{1}$ and $C^{1}$ with the subsemigroups $\overline{B^{1}}=\left\{\left(b, 1_{C}\right): b \in B^{1}\right\}$ and $\overline{C^{1}}=\left\{\left(1_{B}, c\right): c \in C^{1}\right\}$ of $B^{1} \times C^{1}$, respectively. Thus $B^{1}, C^{1} \leq B^{1} \times C^{1}$, and we can consider the actions of $B$ and of $C$ on $A^{B^{1} \times C^{1}}$ involved in the definition of the semidirect products $A$ wr $B\left[B^{1} \times C^{1}\right]$ and $A$ wr $C\left[B^{1} \times C^{1}\right]$, respectively. Consider the semigroup $A$ wr $B\left[B^{1} \times C^{1}\right]$ which we prefer now to denote $A^{B^{1} \times C^{1}} * B$, and define an action of $C$ on $A^{B^{1} \times C^{1}} * B$ 'componentwise', that is, put ${ }^{c}(\alpha, b)=\left({ }^{c} \alpha, b\right)$ for every $c \in C$ and $(\alpha, b) \in A^{B^{1} \times C^{\prime}} * B$. Thus we have defined $\left(A^{B^{\prime} \times C^{1}} * B\right) * C$. 
LeMma 4.8. Let $A, B, C$ be regular semigroups such that $B \in \mathscr{L} \mathscr{Z}$ and $C \in$ $\mathscr{C} \mathscr{S} \backslash \mathscr{G}$.

(i) The mapping $\Theta:\left(A^{B^{1} \times C^{1}} * B\right) * C \rightarrow A \operatorname{wr}(B \times C)\left[B^{1} \times C^{1}\right]$ defined by $((\alpha, b), c) \Theta=(\alpha,(b, c))$ is an isomorphism.

(ii) The subset $\operatorname{Reg}\left(\left(A^{B^{1} \times C^{1}} * B\right) * C\right)$ forms a (regular) subsemigroup in $\left(A^{B^{1} \times C^{1}} * B\right) * C$, we have $\left(A^{B^{1} \times C^{1}} *_{r} B\right) *_{r} C \leq{ }_{r} \operatorname{Reg}\left(\left(A^{B^{1} \times C^{1}} * B\right) * C\right)$, and the mapping $\Theta_{r}: \operatorname{Reg}\left(\left(A^{B^{1} \times C^{1}} * B\right) * C\right) \rightarrow A$ wrr $(B \times C)\left[B^{1} \times C^{1}\right]$ defined by $((\alpha, b), c) \Theta_{r}=(\alpha,(b, c))$ is an isomorphism.

(iii) The restriction of the homomorphism $\Theta_{r} \Upsilon$ to $\left(A^{B^{1} \times C^{1}} *_{r} B\right) *_{r} C$, where $\Upsilon$ is the homomorphism of $A$ wrr $(B \times C)\left[B^{1} \times C^{1}\right]$ onto $A$ wrr $(B \times C)$ given in Remark 3.1, is surjective.

PROOF. (i) The mapping $\Theta$ is obviously bijective. A straightforward calculation shows that it is also a homomorphism. All we have to notice is that ${ }^{b}\left({ }^{c} \alpha\right)={ }^{(b, c)} \alpha$ for every $\alpha \in A^{B^{1} \times C^{\prime}}, b \in B$ and $c \in C$.

(ii) By definition, $\operatorname{Reg}\left(A \operatorname{wr}(B \times C)\left[B^{1} \times C^{1}\right]\right)=A$ wrr $(B \times C)\left[B^{1} \times C^{1}\right]$. Since $\Theta$ and $\Theta^{-1}$ assign regular elements to regular ones, we obtain from (i) that $\operatorname{Reg}\left(\left(A^{B^{I} \times C^{l}} * B\right) * C\right)$ is a (regular) subsemigroup in $\left(A^{B^{I} \times C^{l}} * B\right) * C$ and $\Theta_{r}$ is an isomorphism. Clearly, we have $\left(A^{B^{1} \times C^{1}} *_{r} B\right) *_{r} C \leq_{r} \operatorname{Reg}\left(\left(A^{B^{1} \times C^{1}} * B\right) * C\right)$.

(iii) By (ii) and Remark 3.1, $\Theta_{r} \Upsilon$ is the mapping $\operatorname{Reg}\left(\left(A^{B^{1} \times C^{1}} * B\right) * C\right) \rightarrow$ $A$ wrr $(B \times C),((\alpha, b), c) \mapsto\left(\left.\alpha\right|_{(B \times C)^{1}},(b, c)\right)$, and it is a surjective homomorphism. We have to prove that each element of $A$ wrr $(B \times C)$ has an inverse image in the subsemigroup $\left(A^{B^{1} \times C^{1}} *_{r} B\right) *_{r} C$ of $\operatorname{Reg}\left(\left(A^{B^{1} \times C^{1}} * B\right) * C\right)$. Let $(\gamma,(b, c)) \in$ $A$ wrr $(B \times C)$. Since it is regular, we have $\left(b^{\prime}, c^{\prime}\right) \in V((b, c))$ by Lemma 2.1 such that ${ }^{(b, c)\left(b^{\prime}, c^{\prime}\right)} \gamma \geq \mathscr{L} \gamma$ in $A^{(B \times C)^{1}}$. Here $(b, c)\left(b^{\prime}, c^{\prime}\right)=\left(b, c c^{\prime}\right)$ since $B \in \mathscr{L} \mathscr{Z}$. For brevity, we denote $c c^{\prime}$ by $c^{0}$, and so we have ${ }^{\left(b, c^{0}\right)} \gamma \geq \mathscr{L} \gamma$ in $A^{(B \times C)^{1}}$. The inverse images of $(\gamma,(b, c))$ under $\Theta_{r} \Upsilon$ are the elements $((\alpha, b), c) \in \operatorname{Reg}\left(\left(A^{B^{1} \times C^{1}} * B\right) * C\right)$, where $\alpha \in A^{B^{1} \times C^{\prime}}$ such that $\left.\alpha\right|_{(B \times C)^{1}}=\gamma$. So, in order to reach our goal, we have to extend the definition of $\gamma$ to the set $B^{1} \times C^{1}$ such that the mapping $\alpha$ obtained has the property that $((\alpha, b), c) \in\left(A^{B^{1} \times C^{1}} *_{r} B\right) *_{r} C$, or, equivalently, both $((\alpha, b), c) \in \operatorname{Reg}\left(\left(A^{B^{1} \times C^{1}} * B\right) * C\right)$ and $(\alpha, b) \in \operatorname{Reg}\left(A^{B^{1} \times C^{1}} * B\right)$. By (ii), the former property is equivalent to requiring that $(\alpha,(b, c)) \in \operatorname{Reg}\left(A \operatorname{wr}(B \times C)\left[B^{1} \times C^{1}\right]\right)$, and, by Lemma 2.1, this is satisfied if

$$
{ }^{\left(b, c^{0}\right)} \alpha \geq \mathscr{L} \alpha \quad \text { holds in } \quad A^{B^{1} \times C^{1}} .
$$

Again applying Lemma 2.1 and the fact that $B \in \mathscr{L} \mathscr{Z}$, we see that the latter property is equivalent to the condition that

$$
{ }^{b} \alpha \geq \mathscr{L} \alpha \quad \text { holds in } \quad A^{B^{1} \times C^{1}} .
$$


Define $\alpha: B^{1} \times C^{1} \rightarrow A$ in the following way:

$$
(x, y) \alpha= \begin{cases}(x, y) \gamma & \text { if }(x, y) \in(B \times C)^{1} ; \\ \left(x, c^{0}\right) \gamma & \text { if } x \in B \text { and } y=1_{C} ; \\ (b, y) \gamma & \text { if } x=1_{B} \text { and } y \in C .\end{cases}
$$

Recall that $B \in \mathscr{L} \mathscr{Z}$ and $C \in \mathscr{C} \mathscr{S} \backslash \mathscr{G}$, and therefore neither $B$ nor $C$ has an identity. Hence it follows that $\alpha$ is well defined. We verify $((\alpha, b), c) \in\left(A^{B^{1} \times C^{1}} *_{r} B\right) *_{r} C$ by showing that (4) and (5) are satisfied. If $(x, y) \in(B \times C)^{1}$, then $(x, y)\left({ }^{\left(b, c^{0}\right.} k\right)=$ $\left((x, y)\left(b, c^{0}\right)\right) \alpha=\left((x, y)\left(b, c^{0}\right)\right) \gamma=(x, y)\left({ }^{\left(b, c^{0}\right)} \gamma\right) \geq \mathscr{L}(x, y) \gamma=(x, y) \alpha$. If $x \in$ $B$, then $\left(x, 1_{C}\right)\left({ }^{\left(b, c^{0}\right.}\right)=\left(\left(x, 1_{C}\right)\left(b, c^{0}\right)\right) \alpha=\left(x, c^{0}\right) \alpha=\left(x, c^{0}\right) \gamma=\left(x, 1_{C}\right) \alpha$. Finally, if $y \in C$, then $\left.\left(1_{B}, y\right)\left({ }^{\left(b, c^{0}\right.}\right) \alpha\right)=\left(\left(1_{B}, y\right)\left(b, c^{0}\right)\right) \alpha=\left(b, y c^{0}\right) \alpha=\left(b, y c^{0}\right) \gamma=$ $\left((b, y)\left(b, c^{0}\right)\right) \gamma=(b, y)\left({ }^{\left(b, c^{0}\right)} \gamma\right) \geq \mathscr{L}(b, y) \gamma=\left(1_{B}, y\right) \alpha$. Thus we have shown that (4) is valid. It remains to verify (5). If $x \in B$, then we have $(x, y)\left({ }^{b} \alpha\right)=$ $(x b, y) \alpha=(x, y) \alpha$ for every $y \in C^{1}$. If $y \in C$, then $\left(1_{B}, y\right)\left({ }^{b} \alpha\right)=(b, y) \alpha=$ $(b, y) \gamma=\left(1_{B}, y\right) \alpha$. Finally, we have $\left(1_{B}, 1_{C}\right)\left({ }^{b} \alpha\right)=\left(b, 1_{C}\right) \alpha=\left(b, c^{0}\right) \gamma=$ $\left(1_{B}, 1_{C}\right)\left({ }^{\left(b, c^{0}\right)} \gamma\right) \geq \mathscr{L}\left(1_{B}, 1_{C}\right) \gamma=\left(1_{B}, 1_{C}\right) \alpha$. Thus we have shown that (5) also holds. Hence $((\alpha, b), c) \in\left(A^{B^{1} \times C^{1}} *_{r} B\right) *_{r} C$. Clearly, $\left.\alpha\right|_{(B \times C)^{1}}=\gamma$, and so $((\alpha, b), c) \Theta_{r} \Upsilon=(\gamma,(b, c))$. Thus $A$ wrr $(B \times C)$ is, indeed, a homomorphic image of $\left(A^{B^{1} \times C^{1}} *_{r} B\right) *_{r} C$.

REMARK 4.2. The analogy between the statements of Lemma 4.3 and Lemma 4.4 and the statements of Lemma 4.8 is not by accident: the isomorphisms $\Theta$ and $\Theta_{r}$ can be considered as modifications of the isomorphisms $\Psi$ in Lemma 4.3 and $\Psi_{r}$ in Lemma 4.4, respectively.

More precisely, one can see that the mapping $\Gamma:\left(A^{B^{1} \times C^{1}} * B\right) * C \rightarrow(A$ wr $B)$ wr $C$ defined by $((\alpha, b), c) \Gamma=(\gamma, c)$, where, for every $y \in C^{1}$, we have $y \gamma_{1}: B^{1} \rightarrow A$ such that $x\left(y \gamma_{1}\right)=(x, y) \alpha\left(x \in B^{1}\right)$ and $y \gamma=\left(y \gamma_{1}, b\right)$ is an embedding. The mapping $\Delta: A$ wr $(B \times C)\left[B^{1} \times C^{1}\right] \rightarrow A^{B^{1} \times C^{1}} \star(B$ wr $C)$ given by the rule $(\alpha,(b, c)) \Delta=$ $(\alpha,(\beta, c))$, where $\beta: C^{1} \rightarrow B$ is defined by $y \beta=b\left(y \in C^{1}\right)$ is also an embedding. Denote the images of $\Gamma$ and $\Delta$ by $T$ and $U$, respectively. Denote by $\hat{\Gamma}$ and $\hat{\Delta}$ the isomorphisms of $\left(A^{B^{1} \times C^{1}} * B\right) * C$ onto $T$ and of $A \operatorname{wr}(B \times C)\left[B^{1} \times C^{1}\right]$ onto $U$ induced by $\Gamma$ and by $\Delta$, respectively. It is straightforward to see that the isomorphism $\Psi$ defined in Lemma 4.3 has the property that $T \Psi \subseteq U$ and $U \Psi^{-1} \subseteq T$. Moreover, we also have $\Theta=\hat{\Gamma} \Psi \hat{\Delta}^{-1}$. This shows our assertion for $\Theta$ and $\Psi$. By restricting our considerations to the regular elements, we obtain the respective statement for $\Theta_{r}$ and $\Psi_{r}$.

LEMMA 4.9. Let $\mathscr{U}, \mathscr{V}, \mathscr{W}$ be e-varieties such $\mathscr{V}=\mathscr{L} \mathscr{Z}$ and $\mathscr{W} \subseteq \mathscr{C} \mathscr{S}$ but $\mathscr{W} \nsubseteq \mathscr{G}$. Then $\mathscr{Z}^{\star} \subseteq \mathbf{S}_{r} \mathbf{H S}_{r} \mathscr{Y}$ holds for the classes defined in Corollary 2.1 and Proposition 4.1, respectively. 
PROOF. Let $Z=A^{B^{1} \times C^{1}} \star_{r}(B$ wrr $C)$ be an arbitrary element in $\mathscr{Z}^{\star}$, where $A \in \mathscr{U}$, $B \in \mathscr{V}$ and $C \in \mathscr{W}$. Since $\mathscr{V}=\mathscr{L} \mathscr{Z}$ and $B$ wrr $C$ is a regular semidirect product of the direct power $B^{C^{1}}$ by $C$, we obtain that $B$ wrr $C$ is isomorphic to $B^{C^{1}} \times C$. Since $A^{B^{1} \times C^{1}} \star_{r}\left(B\right.$ wrr $C$ ) is naturally embeddable into $A^{B^{1} \times C^{1}}$ wrr $(B$ wrr $C$ ), we infer that $Z \in \mathbf{S}_{r}\left\{A^{B^{1} \times C^{1}}\right.$ wrr $\left.\left(B^{C^{1}} \times C\right)\right\}$. Since $A^{B^{1} \times C^{1}} \in \mathscr{U}, B^{C^{1}} \in \mathscr{V}$, Lemma 4.8 implies that $Z \in \mathbf{S}, \mathbf{H}\left\{\left(\tilde{A} *_{r} \tilde{B}\right) *_{r} C\right\}$ for some $\tilde{A} \in \mathscr{U}, \tilde{B} \in \mathscr{V}$ and for appropriate actions. Here $\left(\tilde{A} *_{r} \tilde{B}\right) *_{r} C$ is naturally embeddable into $\left(\tilde{A} *_{r} \tilde{B}\right)$ wrr $C$ and $\tilde{A} *_{r} \tilde{B}$ into $\tilde{A}$ wrr $\tilde{B}$. By Lemma 2.3 , we see that $\left(\tilde{A} *_{r} \tilde{B}\right) *_{r} C$ is isomorphic to a regular subsemigroup in $(\tilde{A}$ wrr $\tilde{B})$ wrr $C$. Thus $\left(\tilde{A} *_{r} \tilde{B}\right) *_{r} C \in \mathbf{S}_{r} \mathscr{Y}$, and so $Z \in \mathbf{S}_{r} \mathbf{H} \mathbf{S}_{r} \mathscr{Y}$.

Combining Lemma 4.7 and Lemma 4.9 with Corollary 2.1, Proposition 4.1 and Theorem 4.1, we obtain the main result of this section.

PROPOSITION 4.2. Let $\mathscr{U}, \mathscr{V}, \mathscr{W}$ be e-varieties such that one of the following conditions are satisfied

(i) $\mathscr{U} \subseteq \mathscr{C} \mathscr{S}, \mathscr{V}$ or $\mathscr{W} \subseteq \mathscr{C} \mathscr{S}$ and $\mathscr{V} *_{r} \mathscr{W} \subseteq L \mathscr{I}$ or $\mathscr{E} \mathscr{S}$ (see Result 1.2);

(ii) $\mathscr{V} \subseteq \mathscr{G} \cup \mathscr{L} \mathscr{Z}$ and $\mathscr{W} \subseteq \mathscr{C} \mathscr{S}$;

(iii) $\mathscr{V} \subseteq \mathscr{C} \mathscr{S}$ and $\mathscr{W} \subseteq \mathscr{G}$.

Then $\left(\mathscr{U} *_{r} \mathscr{V}\right) *_{r} \mathscr{W}=\mathscr{U} *_{r}\left(\mathscr{V} *_{r} \mathscr{W}\right)$.

\section{Regular semidirect products of varieties of completely simple semigroups}

In this section we first investigate the regular semidirect products, where one of the factors is a variety of rectangular bands. Then we show that 'almost each' regular semidirect product of completely simple e-varieties is equal to a regular semidirect product, where one of the factors is a group variety. This allows us to generalize Proposition 4.2 by deleting from it the assumption that one of $\mathscr{V}$ and $\mathscr{W}$ lies in a particular subvariety of completely simple semigroups.

First we establish the analogue of Result 1.6 for $\mathscr{R} \mathscr{Z}$.

PROPOSITION 5.1. For any e-variety $\mathscr{V}$, we have $\mathscr{R} \mathscr{Z} * r \mathscr{V}=\mathscr{R} \mathscr{Z} \vee \mathscr{V}$.

ProOF. The inclusion $\mathscr{R} \mathscr{Z} \vee \mathscr{V} \subseteq \mathscr{R} \mathscr{Z} *_{r} \mathscr{V}$ is obvious for any e-variety $\mathscr{V}$. In the sequel we prove the reverse inclusion.

Let $A \in \mathscr{R} \mathscr{Z}, \dot{C} \in \mathscr{V}$, and assume that $C$ acts on $A$. We intend to show that $A * r, C \in \mathscr{R} \mathscr{Z} \vee \mathscr{V}$. By Lemma 2.1, an element $(a, c) \in A * C$ is regular if and only if there exists $c^{\prime} \in V(c)$ such that ${ }^{c c^{\prime}} a \geq \mathscr{L} a$. However, the relation $\geq \mathscr{L}$ is the equality on a right zero semigroup, therefore $(a, c)$ is regular if and only if there exists $c^{\prime} \in V(c)$ such that ${ }^{c c^{\prime}} a=a$. Consider the direct product $A \times C$ and the mapping $\chi: A \times C \rightarrow$ $A * C$ defined by $(a, c) \chi=\left({ }^{c} a, c\right)((a, c) \in A \times C)$. For any $(a, c),(b, d) \in A \times C$, 
we have $(a, c) \chi(b, d) \chi=\left({ }^{c} a, c\right)\left({ }^{d} b, d\right)=\left({ }^{c} a \cdot{ }^{c d} b, c d\right)=\left({ }^{c d} b, c d\right)$ since $A$ is a right zero semigroup. Furthermore, $((a, c)(b, d)) \chi=(a b, c d) \chi=(b, c d) \chi=\left({ }^{c d} b, c d\right)$, and so we see that $\chi$ is a homomorphism. By the observation at the beginning of the proof it is easy to see that $(A \times C) \chi=A *_{r} C$. For, $A \times C$ is a regular semigroup, and so $(A \times C) \chi \subseteq \operatorname{Reg}(A * C)=A *_{r} C$. To verify the reverse inclusion, let $(a, c) \in A *_{r} C$. Then ${ }^{c c^{\prime}} a=a$ for some $c^{\prime} \in V(c)$, and hence $(a, c)=\left({ }^{\prime} a, c\right) \chi$. Clearly, we have $A \times C \in \mathscr{R} \mathscr{Z} \vee \mathscr{V}$, and thus $A *_{r} C \in \mathscr{R} \mathscr{Z} \vee \mathscr{V}$ follows. Hence, by the definition of $\mathscr{R} \mathscr{Z} *_{r} \mathscr{V}$, we obtain that $\mathscr{R} \mathscr{Z} *_{r} \mathscr{V} \subseteq \mathscr{R} \mathscr{Z} \vee \mathscr{V}$.

Since $\mathscr{R} \mathscr{B}=\mathscr{L} \mathscr{Z} \vee \mathscr{R} \mathscr{Z}$, Corollary 2.2, Result 1.6 and Proposition 5.1 imply the following corollary.

\section{COROLLARY 5.1. For any e-variety $\mathscr{V}$, we have $\mathscr{R} \mathscr{B} *_{r} \mathscr{V}=\mathscr{R} \mathscr{B} \vee \mathscr{V}$.}

The regular semidirect products $\mathscr{V} *_{r} \mathscr{R} \mathscr{Z}$ with $\mathscr{V}$ any e-variety are much larger in general than $\mathscr{R} \mathscr{Z} \vee \mathscr{V}$, and they turned out to be especially interesting, see [8,9]. Now we establish that this is not the case with the regular semidirect products of the form $\mathscr{V} *_{r} \mathscr{L} \mathscr{Z}$.

PROPOSITION 5.2. For any e-variety $\mathscr{V}$, we have $\mathscr{V} *_{r} \mathscr{L} \mathscr{Z}=\mathscr{L} \mathscr{Z} \vee \mathscr{V}$.

PROOF. The inclusion $\mathscr{L} \mathscr{Z} \vee \mathscr{V} \subseteq \mathscr{V} * r \mathscr{L} \mathscr{Z}$ is clear by definition. Now we establish the reverse inclusion.

Let $A$ be any regular semigroup and $L$ a left zero semigroup. Define $A_{0}$ to be the left zero semigroup on the set $A$. Define a mapping $\psi: A_{0} \times A^{L} \times L \rightarrow A$ wrr $L$ by the rule $\left(a_{0}, \alpha, e\right) \psi=(\widetilde{\alpha}, e)$, where $1_{L} \widetilde{\alpha}=a_{0} \cdot e \alpha$ and $\left.\tilde{\alpha}\right|_{L}=\alpha$. We intend to verify that $\psi$ is a surjective homomorphism of the direct product of the left zero semigroups $A_{0}$ and $L$ and of the direct power $A^{L}$ of $A$.

By Lemma 2.1, an element $(\beta, f) \in A$ wr $L$ is in $A$ wrr $L$ if and only if ${ }^{f} \beta \geq \mathscr{L} \beta$ in $A^{L^{1}}$. However, observe that, for any $\beta \in A^{L^{1}}$ and $f \in L$, we have $\left.\left({ }^{f} \beta\right)\right|_{L}=\left.\beta\right|_{L}$. For, if $x \in L$, then $x\left({ }^{f} \beta\right)=(x f) \beta=x \beta$. Therefore, $(\beta, f) \in A$ wrr $L$ if and only if $f \beta \geq \mathscr{L} 1_{L} \beta$ in $A$. Since for any $\left(a_{0}, \alpha, e\right) \in A_{0} \times A^{L} \times L$, we have $1_{L} \widetilde{\alpha}=$ $a_{0} \cdot e \alpha \leq \mathscr{L} e \alpha$, this shows that $(\widetilde{\alpha}, e) \in A$ wrr $L$. Conversely, if $(\beta, f) \in A$ wrr $L$, then $f \beta \geq \mathscr{L} 1_{L} \beta$ in $A$ which implies that $1_{L} \beta=u \cdot f \beta$ holds for some $u \in A$. Thus $\left(u,\left.\beta\right|_{L}, f\right) \psi=(\beta, f)$, proving that $\psi$ is a surjective mapping onto $A$ wrr $L$.

In order to verify that it is a homomorphism, consider $\left(a_{0}, \alpha, e\right),\left(b_{0}, \beta, f\right) \in$ $A_{0} \times A^{L} \times L$. Then

$$
\left(\left(a_{0}, \alpha, e\right)\left(b_{0}, \beta, f\right)\right) \psi=\left(a_{0}, \alpha \beta, e\right) \psi=(\gamma, e),
$$

where $1_{L} \gamma=a_{0} \cdot e(\alpha \beta)$ and $\left.\gamma\right|_{L}=\alpha \beta$. Furthermore, we have

$$
\left(a_{0}, \alpha, e\right) \psi \cdot\left(b_{0}, \beta, f\right) \psi=(\widetilde{\alpha}, e)(\widetilde{\beta}, f)=(\widetilde{\alpha} \cdot e \widetilde{\beta}, e),
$$


where $1_{L} \tilde{\alpha}=a_{0} \cdot e \alpha, 1_{L} \widetilde{\beta}=b_{0} \cdot f \beta,\left.\widetilde{\alpha}\right|_{L}=\alpha$ and $\left.\widetilde{\beta}\right|_{L}=\beta$. Thus

$$
1_{L} \gamma=a_{0} \cdot e(\alpha \beta)=a_{0} \cdot e \alpha \cdot e \beta=1_{L} \tilde{\alpha} \cdot e \widetilde{\beta}=1_{L}(\widetilde{\alpha} \cdot e \widetilde{\beta})
$$

and, for any $x \in L$,

$$
x \gamma=x(\alpha \beta)=x \alpha \cdot x \beta=x \alpha \cdot(x e) \beta=x \widetilde{\alpha} \cdot(x e) \widetilde{\beta}=x\left(\widetilde{\alpha} \cdot e^{e} \widetilde{\beta}\right) .
$$

Thus we have checked that $\psi$ is also a homomorphism.

If $A \in \mathscr{V}$ and $L \in \mathscr{L} \mathscr{Z}$, then $A_{0} \times A^{L} \times L \in \mathscr{L} \mathscr{Z} \vee \mathscr{V}$. The assertion in the previous paragraph shows that $A$ wrr $L \in \mathscr{L} \mathscr{Z} \vee \mathscr{V}$. By Result 1.1 we infer that $\mathscr{V} *_{r} \mathscr{L} \mathscr{Z} \subseteq \mathscr{L} \mathscr{Z} \vee \mathscr{V}$, and the proof is complete.

COROLLARY 5.2. For any e-variety $\mathscr{V}$ with $\mathscr{V} \nsubseteq \mathscr{R} \mathscr{Z}$, we have $\mathscr{V} *_{r} \mathscr{R} \mathscr{B}=$ $\mathscr{V} *_{r} \mathscr{R} \mathscr{Z}$.

PROOF. Since we have $\mathscr{R} \mathscr{B}=\mathscr{L} \mathscr{Z} *_{r} \mathscr{R} \mathscr{Z}$ by Result 1.6, Proposition 4.2 implies that $\mathscr{V} *_{r} \mathscr{R} \mathscr{B}=\left(\mathscr{V} *_{r} \mathscr{L} \mathscr{Z}\right) *_{r} \mathscr{R} \mathscr{Z}$. By applying Proposition 5.2, Corollary 2.2 and Result 1.6, we see that

$$
\begin{aligned}
\left(\mathscr{V} *_{r} \mathscr{L} \mathscr{Z}\right) *_{r} \mathscr{R} \mathscr{Z} & =(\mathscr{L} \mathscr{Z} \vee \mathscr{V}) *_{r} \mathscr{R} \mathscr{Z}=\left(\mathscr{L} \mathscr{Z} *_{r} \mathscr{R} \mathscr{Z}\right) \vee\left(\mathscr{V} *_{r} \mathscr{R} \mathscr{Z}\right) \\
& =\mathscr{R} \mathscr{B} \vee\left(\mathscr{V}_{r} \mathscr{R} \mathscr{Z}\right) .
\end{aligned}
$$

Result 1.4 (ii) ensures that $\mathscr{V} *_{r} \mathscr{R} \mathscr{Z} \supseteq \mathscr{L} \mathscr{Z}$ whence $\mathscr{V} *_{r} \mathscr{R} \mathscr{Z} \supseteq \mathscr{R} \mathscr{B}$ follows. Thus we infer that $\mathscr{V} *_{r} \mathscr{R} \mathscr{B}=\mathscr{V} *_{r} \mathscr{R} \mathscr{Z}$.

In the following two propositions we provide regular semidirect products which are equal to regular semidirect products one of whose factors is a variety of groups. As a corollary, we establish that 'almost always' this is the case with regular semidirect products of varieties of completely simple semigroups.

PROPOSITION 5.3. Let $\mathscr{V}, \mathscr{W}$ be e-varieties such that $\mathscr{V} \subseteq \mathscr{C} \mathscr{S}$ and $\mathscr{R} \mathscr{Z} \subseteq \mathscr{W} \subseteq$ $L \mathscr{I}$ or $\mathscr{E} \mathscr{S}$. Then we have

$$
\mathscr{V} *_{r} \mathscr{W}= \begin{cases}(\mathscr{V} \cap \mathscr{G}) *_{r} \mathscr{W} & \text { if } \mathscr{V} \cap \mathscr{G} \neq \mathscr{T} ; \\ (\mathscr{V} \cap \mathscr{L} \mathscr{Z}) \vee \mathscr{W} & \text { otherwise. }\end{cases}
$$

PRoOF. By Proposition 5.1, we have $\mathscr{W}=\mathscr{R} \mathscr{Z} *_{r} \mathscr{W}$. Therefore, by Proposition 4.2 , we see that

$$
\mathscr{V} *_{r} \mathscr{W}=\mathscr{V} *_{r}\left(\mathscr{R} \mathscr{Z} *_{r} \mathscr{W}\right)=\left(\mathscr{V} *_{r} \mathscr{R} \mathscr{Z}\right) *_{r} \mathscr{W}
$$


Suppose first that $\mathscr{V} \cap \mathscr{G} \neq \mathscr{T}$. Result 1.4 (i) implies that

$$
(\mathscr{V} \cap \mathscr{G}) *_{r} \mathscr{R} \mathscr{Z} \subseteq \mathscr{V} *_{r} \mathscr{R} \mathscr{Z} \subseteq L \mathscr{V}
$$

However, completely simple semigroups are just the regular semigroups whose local submonoids are groups. Therefore, we have

$$
L \mathscr{V}=L(\mathscr{V} \cap \mathscr{G})=\mathscr{C} \mathscr{S}(\mathscr{V} \cap \mathscr{G})
$$

Applying Result 1.4 (ii), we see that

$$
\mathscr{C} \mathscr{S}(\mathscr{V} \cap \mathscr{G})=(\mathscr{V} \cap \mathscr{G}) *, \mathscr{P} \mathscr{Z} .
$$

Hence $\mathscr{V} *_{r} \mathscr{R} \mathscr{Z}=(\mathscr{V} \cap \mathscr{G}) *_{r} \mathscr{R} \mathscr{Z}$ follows. Thus we obtain that

$$
\begin{aligned}
\mathscr{V} *_{r} \mathscr{W} & =\left(\mathscr{V} *_{r} \mathscr{R} \mathscr{Z}\right) *_{r} \mathscr{W}=\left((\mathscr{V} \cap \mathscr{G}) *_{r} \mathscr{R} \mathscr{Z}\right) *_{r} \mathscr{W} \\
& =(\mathscr{V} \cap \mathscr{G}) *_{r}\left(\mathscr{R} \mathscr{Z} *_{r} \mathscr{W}\right)=(\mathscr{V} \cap \mathscr{G}) *_{r} \mathscr{W} .
\end{aligned}
$$

In the last two steps we again applied Proposition 4.2 and Proposition 5.1.

If $\mathscr{V} \cap \mathscr{G}=\mathscr{T}$, then $\mathscr{V} \subseteq \mathscr{R} \mathscr{B}$. Since the subvarieties in $\mathscr{R} \mathscr{B}$ are just $\mathscr{T}, \mathscr{L} \mathscr{Z}, \mathscr{R} \mathscr{Z}$ and $\mathscr{R} \mathscr{B}$, the trivial equality $\mathscr{T} *_{r} \mathscr{V}=\mathscr{V}$, Result 1.6, Proposition 5.1 and Corollary 5.1, respectively, imply that $\mathscr{V} *_{r} \mathscr{W}=\mathscr{V} \vee \mathscr{W}$. Since $\mathscr{R} \mathscr{Z} \subseteq \mathscr{W}$, we have $\mathscr{V} \vee \mathscr{W}=(\mathscr{V} \cap \mathscr{L} \mathscr{Z}) \vee(\mathscr{V} \cap \mathscr{R} \mathscr{Z}) \vee \mathscr{W}=(\mathscr{V} \cap \mathscr{L} \mathscr{Z}) \vee \mathscr{W}$, completing the proof.

PROPOSITION 5.4. Let $\mathscr{V}, \mathscr{W}$ be e-varieties such that $\mathscr{W} \subseteq \mathscr{L} \mathscr{G}$. Then we have $\mathscr{V} *_{r} \mathscr{W}=(\mathscr{V} \vee(\mathscr{W} \cap \mathscr{L} \mathscr{Z})) *_{r}(\mathscr{W} \cap \mathscr{G})$.

PROOF. If $\mathscr{W} \subseteq \mathscr{G}$, then the equality is trivial. In the opposite case, we have $\mathscr{L} \mathscr{Z} \subseteq \mathscr{W}$, and so the equality to be proved is $\mathscr{V} *_{r} \mathscr{W}=(\mathscr{V} \vee \mathscr{L} \mathscr{Z}) *_{r}(\mathscr{W} \cap \mathscr{G})$. Since $\mathscr{W}=\mathscr{L} \mathscr{Z} \vee(\mathscr{W} \cap \mathscr{G})$, Result 1.6, Proposition 4.2 and Proposition 5.2 imply that

$$
\begin{aligned}
\mathscr{V} *_{r} \mathscr{W} & =\mathscr{V} *_{r}(\mathscr{L} \mathscr{Z} \vee(\mathscr{W} \cap \mathscr{G}))=\mathscr{V} *_{r}\left(\mathscr{L} \mathscr{Z} *_{r}(\mathscr{W} \cap \mathscr{G})\right) \\
& =\left(\mathscr{V} *_{r} \mathscr{L} \mathscr{Z}\right) *_{r}(\mathscr{W} \cap \mathscr{G})=(\mathscr{V} \vee \mathscr{L} \mathscr{Z}) *_{r}(\mathscr{W} \cap \mathscr{G}) .
\end{aligned}
$$

Now let $\mathscr{V}, \mathscr{W}$ be subvarieties in $\mathscr{C} \mathscr{S}$. Since we have either $\mathscr{W} \supseteq \mathscr{R} \mathscr{Z}$ or $\mathscr{W} \subseteq \mathscr{L} \mathscr{G}$, Proposition 5.3 and Proposition 5.4 imply the following result.

COROLLARY 5.3. Let $\mathscr{V}, \mathscr{W}$ be varieties of completely simple semigroups. Then we have

$$
\mathscr{V} *_{r} \mathscr{W}= \begin{cases}(\mathscr{V} \cap \mathscr{G}) *_{r} \mathscr{W} & \text { if } \mathscr{V} \nsubseteq \mathscr{R} \mathscr{B} \text { and } \mathscr{R} \mathscr{Z} \subseteq \mathscr{W} \\ (\mathscr{V} \cap \mathscr{L} \mathscr{Z}) \vee \mathscr{W} & \text { if } \mathscr{V} \subseteq \mathscr{R} \mathscr{B} \text { and } \mathscr{R} \mathscr{Z} \subseteq \mathscr{W} \\ (\mathscr{V} \vee(\mathscr{W} \cap \mathscr{L} \mathscr{Z})) *_{r}(\mathscr{W} \cap \mathscr{G}) & \text { if } \mathscr{W} \subseteq \mathscr{L} \mathscr{G}\end{cases}
$$


Finally, we return to investigate associativity of the regular semidirect product, and prove the main result of the paper.

THEOREM 5.1. Let $\mathscr{U}, \mathscr{V}, \mathscr{W}$ be e-varieties such that one of the following conditions are satisfied

(i) $\mathscr{U} \subseteq \mathscr{C} \mathscr{S}, \mathscr{V}$ or $\mathscr{W} \subseteq \mathscr{C} \mathscr{S}$ and $\mathscr{V} *_{r} \mathscr{W} \subseteq L \mathscr{I}$ or $\mathscr{E} \mathscr{S}$

(ii) $\mathscr{V}, \mathscr{W} \subseteq \mathscr{C} \mathscr{S}$.

Then $\left(\mathscr{U} *_{r} \mathscr{V}\right) *_{r} \mathscr{W}=\mathscr{U} *_{r}\left(\mathscr{V} *_{r} \mathscr{W}\right)$.

REMARK 5.1. Result 1.2 makes it possible to formulate condition (i) by posing restrictions on the e-varieties $\mathscr{U}, \mathscr{V}, \mathscr{W}$ only. Thus (i) is equivalent to requiring that (i) $\mathscr{U} \subseteq \mathscr{C} \mathscr{S}$, and $\mathscr{V}, \mathscr{W}$ fulfil one of the condition (LI1), (LI2), (ES1) and (ES2).

PROOF. Taking into account Proposition 4.2, we have to prove only (ii) in the case, where $\mathscr{U} \nsubseteq \mathscr{C} \mathscr{S}, \mathscr{V} \nsubseteq \mathscr{G} \cup \mathscr{L} \mathscr{Z}$ and $\mathscr{W} \nsubseteq \mathscr{G}$. Moreover, by Theorem 4.1, it suffices to verify the inclusion $\mathscr{U} *_{r}\left(\mathscr{V} *_{r} \mathscr{W}\right) \subseteq\left(\mathscr{U} *_{r} \mathscr{V}\right) *_{r} \mathscr{W}$. We distinguish three cases according to those listed in Corollary 5.3.

If $\mathscr{V} \nsubseteq \mathscr{R} \mathscr{B}$ and $\mathscr{R} \mathscr{Z} \subseteq \mathscr{W}$, then we have

$$
\begin{aligned}
\mathscr{U} *_{r}\left(\mathscr{V} *_{r} \mathscr{W}\right) & =\mathscr{U} *_{r}\left((\mathscr{V} \cap \mathscr{G}) *_{r} \mathscr{W}\right) \\
& =\left(\mathscr{U} *_{r}(\mathscr{V} \cap \mathscr{G})\right) *_{r} \mathscr{W} \quad \text { by Proposition } 4.2 \\
& \subseteq\left(\mathscr{U} *_{r} \mathscr{V}\right) *_{r} \mathscr{W} .
\end{aligned}
$$

If $\mathscr{V} \subseteq \mathscr{R} \mathscr{B}$ and $\mathscr{R} \mathscr{Z} \subseteq \mathscr{W}$, then we have $\mathscr{U} *_{r}\left(\mathscr{V} *_{r} \mathscr{W}\right)=\mathscr{U} *_{r}((\mathscr{V} \cap$ $\mathscr{L} \mathscr{Z}) \vee \mathscr{W})$. Here $\mathscr{V} \cap \mathscr{L} \mathscr{Z}=\mathscr{T}$ or $\mathscr{L} \mathscr{Z}$. If $\mathscr{V} \cap \mathscr{L} \mathscr{Z} \subseteq \mathscr{W}$, then we obtain $\mathscr{U} *_{r}\left(\mathscr{V} *_{r} \mathscr{W}\right)=\mathscr{U} *_{r} \mathscr{W} \subseteq\left(\mathscr{U} *_{r} \mathscr{V}\right) *_{r} \mathscr{W}$. If $\mathscr{V} \cap \mathscr{L} \mathscr{Z} \nsubseteq \mathscr{W}$, then we necessarily have $\mathscr{V} \cap \mathscr{L} \mathscr{Z}=\mathscr{L} \mathscr{Z}$ and $\mathscr{W} \subseteq \mathscr{R} \mathscr{G}$. The latter relation together with $\mathscr{R} \mathscr{Z} \subseteq \mathscr{W}$ ensures that $\mathscr{W}=\mathscr{R} \mathscr{Z} \vee \mathscr{K}$, where $\mathscr{K}=\mathscr{W} \cap \mathscr{G}$. Thus we have

$$
\begin{aligned}
\mathscr{U} *_{r}\left(\mathscr{V} *_{r} \mathscr{W}\right) & =\mathscr{U} *_{r}(\mathscr{L} \mathscr{Z} \vee \mathscr{R} \mathscr{Z} \vee \mathscr{K}) \\
& =\mathscr{U} *_{r}\left(\mathscr{R} \mathscr{B} *_{r} \mathscr{K}\right) \quad \text { by Corollary } 5.1 \\
& =\left(\mathscr{U} *_{r} \mathscr{R} \mathscr{B}\right) *_{r} \mathscr{K} \quad \text { by Proposition } 4.2 \\
& =\left(\mathscr{U} *_{r} \mathscr{R} \mathscr{Z}\right) *_{r} \mathscr{K} \quad \text { by Corollary } 5.2, \text { since } \mathscr{U} \nsubseteq \mathscr{C} \mathscr{S} \\
& =\mathscr{U} *_{r}\left(\mathscr{R} \mathscr{Z} *_{r} \mathscr{K}\right) \quad \text { by Proposition } 4.2 \\
& =\mathscr{U} *_{r} \mathscr{W} \quad \text { by Proposition } 5.1 \\
& \subseteq\left(\mathscr{U} *_{r} \mathscr{V}\right) *_{r} \mathscr{W} .
\end{aligned}
$$

Finally, let $\mathscr{W} \subseteq \mathscr{L} \mathscr{G}$. Since $\mathscr{W} \nsubseteq \mathscr{G}$, we have $\mathscr{W}=\mathscr{L} \mathscr{Z} \vee \mathscr{K}$, where $\mathscr{K}=\mathscr{W} \cap \mathscr{G}$. Then Corollary 5.3 implies that $\mathscr{V} *_{r} \mathscr{W}=(\mathscr{V} \vee \mathscr{L} \mathscr{Z}) *_{r} \mathscr{K}$. In 
particular, if $\mathscr{L} \mathscr{Z} \subseteq \mathscr{V}$, then we see by Proposition 4.2 that

$$
\mathscr{U} *_{r}\left(\mathscr{V} *_{r} \mathscr{W}\right)=\mathscr{U} *_{r}\left(\mathscr{V} *_{r} \mathscr{K}\right)=\left(\mathscr{U} *_{r} \mathscr{V}\right) *_{r} \mathscr{K} \subseteq\left(\mathscr{U} *_{r} \mathscr{V}\right) *_{r} \mathscr{W}
$$

If $\mathscr{L} \mathscr{Z} \nsubseteq \mathscr{V}$, then $\mathscr{V} \subseteq \mathscr{R} \mathscr{G}$ and, since $\mathscr{V} \nsubseteq \mathscr{G}$, we have $\mathscr{V}=\mathscr{R} \mathscr{Z} \vee \mathscr{M}$, where $\mathscr{M}=\mathscr{V} \cap \mathscr{G}$. Therefore,

$$
\begin{aligned}
& \mathscr{U} *_{r}\left(\mathscr{V} *_{r} \mathscr{W}\right)=\mathscr{U} *_{r}\left((\mathscr{L} \mathscr{Z} \vee \mathscr{R} \mathscr{Z} \vee \mathscr{M}) *_{r} \mathscr{K}\right) \\
& =\mathscr{U} *_{r}\left(\left(\mathscr{R} \mathscr{B} *_{r} \mathscr{M}\right) *_{r} \mathscr{K}\right) \quad \text { by Corollary } 5.1 \\
& =\mathscr{U} *_{r}\left(\mathscr{R} \mathscr{B} *_{r}\left(\mathscr{H} *_{r} \mathscr{K}\right)\right) \quad \text { by Proposition } 4.2 \\
& =\left(\mathscr{U} *_{r} \mathscr{R} \mathscr{B}\right) *_{r}\left(\mathscr{M} *_{r} \mathscr{K}\right) \quad \text { by Proposition } 4.2 \\
& =\left(\mathscr{U} *_{r} \mathscr{R} \mathscr{Z}\right) *_{r}\left(\mathscr{M} *_{r} \mathscr{K}\right) \quad \text { by Corollary } 5.2 \text {, since } \mathscr{U} \nsubseteq \mathscr{C} \mathscr{S} \\
& =\left(\left(\mathscr{U} *_{r} \mathscr{R} \mathscr{Z}\right) *_{r} \mathscr{M}\right) *_{r} \mathscr{K} \quad \text { by Proposition } 4.2 \\
& =\left(\mathscr{U} *_{r}\left(\mathscr{R} \mathscr{Z} *_{r} \mathscr{M}\right)\right) *_{r} \mathscr{K} \quad \text { by Proposition } 4.2 \\
& =\left(\mathscr{U} *_{r} \mathscr{V}\right) *_{r} \mathscr{K} \quad \text { by Proposition } 5.1 \\
& \subseteq\left(\mathscr{U} *_{r} \mathscr{V}\right) *_{r} \mathscr{W}
\end{aligned}
$$

By Result 1.5, the formation of a regular semidirect product is a full operation on the set of all varieties of completely simple semigroups. Thus we obtain the following corollary.

COROLLARY 5.4. On the complete lattice of all varieties of completely simple semigroups, the regular semidirect product is an associative operation, and it is right distributive with respect to the join operation.

\section{Regular semidirect products of e-pseudovarieties}

In this section we review the former arguments from the point of view how they carry over to e-pseudovarieties of finite regular semigroups. We find that both right distributivity with respect to the join operation and associativity hold in the complete lattice of e-pseudovarieties.

The finitary analogue of the concept of an e-variety is the following (see [12]): an e-pseudovariety is a class of finite regular semigroups which is closed under the formation of finite direct products, regular subsemigroups and homomorphic images. Notice that the class of all finite members of an e-variety forms an e-pseudovariety.

Throughout this section, the notation of the previous sections is used with the appropriate modification 'cut down to the finite case' in its meaning. In particular, a notation used till now for an e-variety will stand for the e-pseudovariety of all of its 
finite members. For example, $\mathscr{C} \mathscr{S}$ denotes the e-pseudovariety of all finite completely simple semigroups and $\mathscr{C} \mathscr{S}(\mathscr{V})$ its sub-e-pseudovariety consisting of all members whose subgroups belong to a given e-pseudovariety $\mathscr{V}$ of finite groups. Moreover, if $\mathscr{C}$ is a class of finite regular semigroups, then $\langle\mathscr{C}\rangle_{e}$ is understood as the e-pseudovariety generated by $\mathscr{C}$ and $\mathbf{P} \mathscr{C}$ as the class of all finite direct products of members of $\mathscr{C}$.

In the context of e-pseudovarieties, the difficulty mentioned in Section 1 in connection with expressing the generated e-variety by means of the operators $\mathbf{D}^{\infty}$ and $\mathbf{P}$ does not occur: we have $\langle\mathscr{C}\rangle_{e}=\mathbf{D}^{\infty} \mathbf{P} \mathscr{C}$ for every class $\mathscr{C}$ of finite regular semigroups ([12]). This is the reason that more general results are obtained for e-pseudovarieties than for e-varieties.

The definition of the regular semidirect product $*_{r}$ of e-varieties carries over immediately to e-pseudovarieties. As it was noticed in [1], Result 1.1-Result 1.5 remain valid in the latter context. One can easily see that the same is the case with Result 1.6 and with the results in Section 2. In particular, the finitary analogue of Corollary 2.2 formulates the right distributivity of the regular semidirect product of e-pseudovarieties with respect to their join.

COROLLARY 6.1. Let $\mathscr{U}_{i}(i \in I)$ and $\mathscr{V}$ be e-pseudovarieties such that either $\mathscr{U}_{i} \subseteq$ $\mathscr{C} \mathscr{S}$ for each $i \in I$ or $\mathscr{V} \subseteq \mathscr{C} \mathscr{S}$. Then we have $\left(\bigvee_{i \in I} \mathscr{U}_{i}\right) *_{r} \mathscr{V}=\bigvee_{i \in I}\left(\mathscr{U}_{i} *_{r} \mathscr{V}\right)$

Similarly, one can check that the statement of Theorem 3.1 is also valid for epseudovarieties instead of e-varieties.Since, for e-pseudovarieties, the equality $\mathscr{V}=$ $\mathbf{D}^{\infty} \mathscr{X}$ is equivalent to requiring that $\mathscr{V}=\langle\mathscr{X}\rangle_{e}$ and $\mathbf{P} \mathscr{X} \subseteq \mathbf{D}^{\infty} \mathscr{X}$, the analogue of Corollary 3.1 is the following corollary.

COROLlaRY 6.2. Let $\mathscr{U}, \mathscr{V}$ be e-pseudovarieties such that $\mathscr{U}$ or $\mathscr{V} \subseteq \mathscr{C} \mathscr{S}$. Let $\mathscr{X} \subseteq \mathscr{V}$ such that $\mathscr{V}=\langle\mathscr{X}\rangle_{e}$ and $\mathbf{P} \mathscr{X} \subseteq \mathbf{D}^{\infty} \mathscr{X}$. Then $\mathscr{U} *_{r} \mathscr{V}=\langle A$ wrr $X$ : $A \in \mathscr{U}, X \in \mathscr{X}\rangle_{\text {e. }}$

Hence the argument after Corollary 3.1 implies the following statement.

COROLLARY 6.3. Let $\mathscr{U}, \mathscr{V}, \mathscr{W}$ be e-pseudovarieties such that at least two of them are contained in $\mathscr{C} \mathscr{S}$. Then $\mathscr{U} *_{r}\left(\mathscr{V} *_{r} \mathscr{W}\right)$ is defined, and it is generated by

$$
\mathscr{Z}=\{A \text { wrr }(B \text { wrr } C): A \in \mathscr{U}, B \in \mathscr{V}, C \in \mathscr{W}\}
$$

Taking into account the symmetry of Corollary 6.3 and of the finitary analogue of Corollary 2.1 , the arguments in Section 4 prove the equality $\left(\mathscr{U} *_{r} \mathscr{V}\right) *_{r} \mathscr{W}=$ $\mathscr{U} *_{r}\left(\mathscr{V} *_{r} \mathscr{W}\right)$ for any e-pseudovarieties $\mathscr{U}, \mathscr{V}, \mathscr{W}$, where either (i) $\mathscr{U} \subseteq \mathscr{C} \mathscr{S}$ and one of $\mathscr{V}, \mathscr{W}$ is in $\mathscr{C} \mathscr{S}$, or (ii) $\mathscr{V} \subseteq \mathscr{G} \cup \mathscr{L} \mathscr{Z}$ and $\mathscr{W} \subseteq \mathscr{C} \mathscr{S}$, or (iii) $\mathscr{V} \subseteq \mathscr{C} \mathscr{S}$ and $\mathscr{W} \subseteq \mathscr{G}$ (see Proposition 4.2). 
Finally, since the basic statements on the e-varieties of rectangular bands and of left and right groups applied in Section 5 (for example, all the sub-e-pseudovarieties of $\mathscr{R} \mathscr{B}$ are $\mathscr{T}, \mathscr{L} \mathscr{Z}, \mathscr{R} \mathscr{Z}$ and $\mathscr{R} \mathscr{B}$; each sub-e-pseudovariety $\mathscr{V}$ of $\mathscr{L} \mathscr{G}$ is of the form $\mathscr{L} \mathscr{Z} \vee \mathscr{K}$, where $\mathscr{K}=\mathscr{V} \cap \mathscr{G}$ ) are valid in the context of e-pseudovarieties, the arguments in Section 5 verify the associativity of the regular semidirect product of e-pseudovarieties.

THEOREM 6.1. Let $\mathscr{U}, \mathscr{Y}, \mathscr{W}$ be e-pseudovarieties such that at least two of them are contained in $\mathscr{C} \mathscr{S}$. Then $\left(\mathscr{U} *_{r} \mathscr{V}\right) *_{r} \mathscr{W}=\mathscr{U} *_{r}\left(\mathscr{V} *_{r} \mathscr{W}\right)$.

\section{Acknowledgement}

Research partially supported by the Hungarian National Foundation for Scientific Research grants no. T22867 and T26243 and by the Ministry of Education grant no. FKFP 1030/1997. The result was obtained while the second author was a Humboldt fellow at the FB Mathematik/Informatik, Universität Gesamthochschule Kassel. The excellent conditions provided by the fellowship and by the GhK are gratefully acknowledged.

\section{References}

[1] K. Auinger and P. G. Trotter, 'Pseudovarieties, regular semigroups and semidirect products', J. London Math. Soc. (2) 58 (1998), 284-296.

[2] B. Billhardt, 'On $\lambda$-semidirect products by locally $\mathscr{R}$-unipotent semigroups', Acta Sci. Math., to appear.

[3] —-, 'On a wreath product embedding and idempotent pure congruences on inverse semigroups', Semigroup Forum 45 (1992), 45-54.

[4] S. Eilenberg, Automata, languages and machines, Vol. B (Academic Press, Berlin, 1976).

[5] T. E. Hall, 'Identities for existence varieties of regular semigroups', Bull. Austral. Math. Soc. 40 (1989), 59-77.

[6] C. H. Houghton, 'Embedding inverse semigroups in wreath products', Glasgow Math. J. 17 (1976), 77-82.

[7] J. M. Howie, Fundamentals of semigroup theory, London Math. Soc. Monographs, New Series 12 (Oxford University Press, Oxford, 1995).

[8] P. R. Jones, 'Rees matrix covers and semidirect products of regular semigroups', J. Algebra 218 (1999), 287-306.

[9] P. R. Jones and P. G. Trotter, 'Semidirect products of regular semigroups', Trans. Amer. Math. Soc. 349 (1997), 4265-4310.

[10] J. Kad'ourek, 'On some existence varieties of locally orthodox semigroups', Internat. J. Algebra Comput. 7 (1997), 93-131.

[11] J. Kad'ourek and M. B. Szendrei, 'A new approach in the theory of orthodox semigroups', Semigroup Forum 40 (1990), 257-296. 
[12] M. Mangold, E-varieties and e-pseudovarieties of regular semigroups (Ph.D. Thesis, Monash University, 1995).

[13] J. D. P. Meldrum, Wreath products of groups and semigroups, Pitman Monographs Surveys Pure Appl. Math. 74 (Longman, Essex, 1995).

[14] H. Neumann, Varieties of groups (Springer, Berlin, 1967).

[15] N. R. Reilly and S. Zhang, 'Associativity of products of existence varieties of regular semigroups', J. Pure Appl. Algebra 135 (1999), 57-91.

[16] Y. T. Yeh, 'The existence of e-free objects in e-varieties of regular semigroups', Internat. J. Algebra Comput. 2 (1992), 471-484.

Universität-Gesamthochschule Kassel

Fachbereich 17 Mathematik/Informatik

D-34109 Kassel

Holländische Str. 36

Germany

e-mail: billard@hrz.uni-kassel.de
University of Szeged

Bolyai Institute

H-6720 Szeged

Aradi vértanúk tere 1

Hungary

e-mail: M.Szendrei@math.u-szeged.hu 